\title{
1 Invariances in a combinatorial olfactory receptor code
}

3 Guangwei Si $\left(1,2,{ }^{*}\right)$, Jessleen K. Kanwal $\left(2,3,{ }^{*}\right)$, Yu Hu (2), Christopher J. Tabone (1, 2),

4 Jacob Baron (1, 2), Matthew Berck (1, 2), Gaetan Vignoud (1, 2), Aravinthan D.T. Samuel (1, 2 , $5+)$

6

7 1. Department of Physics, Harvard University, Cambridge, MA 02138

8 2. Center for Brain Science, Harvard University, Cambridge, MA 02138

9 3. Program in Neuroscience, Harvard University, Cambridge, MA 02138

10 * Equal contributions

11 † To whom correspondence should be addressed 


\section{Abstract}

14 Animals can identify an odorant type across a wide range of concentrations, as well as detect changes in concentration for individual odorant type. How olfactory representations are structured to support these functions remains poorly understood.

17 Here, we studied how a full complement of ORNs in the Drosophila larva encodes a 18 broad input space of odorant types and concentrations. We find that dose-response 19 relationships across odorants and ORN types follow the Hill function with shared cooperativity but different activation thresholds. These activation thresholds are drawn

21 from a power law statistical distribution. A fixed activation function and power law

22 distribution of activation thresholds underlie invariances in the encoding of odorant

23 identity and intensity. Moreover, we find similar temporal response filters of ORNs

24 across odorant types and concentrations. Such uniformity in the temporal filter may

25 allow identity invariant coding in fluctuating or turbulent odor environments. Common patterns in ligand-receptor binding and sensory transduction across olfactory receptors may give rise to these observed invariances in the olfactory combinatorial code. Invariant patterns in the activity responses of individual ORNs and the ORN ensemble may simplify decoding by downstream circuits. 


\section{Introduction}

32 The abilities to identify odorants across a wide range of concentrations and detect changes in odorant concentration are essential for olfactory perception and behavior. Olfactory systems use combinatorial codes to encode large numbers of odors with smaller numbers of olfactory receptor neurons (ORNs) (Malnic et al., 1999). Each ORN typically expresses one of a large repertoire of olfactory receptors (Ors) (Buck and Axel, 1991). A single Or can be activated by many different odorants, and a single odorant can activate many different Ors (Friedrich and Korsching, 1997). Different odorants can be discriminated by distinct activity patterns across an ensemble of olfactory neurons (Hallem and Carlson, 2006; Kreher et al., 2005; Nara et al., 2011). The olfactory code also conveys information about odorant intensity as higher odorant concentrations tend to activate more ORNs (Kajiya et al., 2001; Wang et al., 2003). Different odorants may also evoke different temporal patterns in neuronal activity, augmenting information coding using time (Friedrich and Laurent, 2001; Laurent et al., 2001; Junek et al., 2010; Smear et al., 2011).

Recent studies have uncovered coding properties at the single cell and population levels that may allow for scale-invariant representation of olfactory information such as odorant type and intensity. At the individual ORN level, ORN responses to temporal patterns in odorant presentation may be converted into predictable activity patterns by stereotyped filters (Nagel and Wilson, 2011; Martelli et al., 2013). At the population level, inputs to the olfactory bulb may encode odorants in concentration invariant spatial representations (Wachowiak et al., 2002; Cleland et al., 2007). At the statistical level, the firing rates of Drosophila ORNs appear to be drawn from an odor invariant probability distribution (Stevens, 2016). However, a quantitative characterization of such invariances in olfactory representation by a complete ORN ensemble is still missing.

In this study, we characterized the ORN ensemble of the Drosophila larva to a panel of odorant types and concentrations that spanned the selectivity of all olfactory sensory neurons. The Drosophila larva offers the advantage of numerical simplicity for dissecting an olfactory circuit that shares glomerular organization with adult insects and vertebrates (Vosshall and Stocker,

61 2007; Su et al., 2009). We find that ORN-odorant pairs share the same activation function: ORN

62 activity increases with concentration along the same Hill curve for any odorant type but with 63 odorant-specific thresholds. We find that the statistical distribution of these ORN sensitivities to 64 odorants across olfactory space follows a power-law. Furthermore, ORNs share a stereotyped 
temporal filter shape such that ensemble level responses may be concentration-invariant in a fluctuating environment. Our systems-level characterization of an entire olfactory periphery across a wide range of odorant types and concentrations has revealed individual and ensemble level ORN patterns that allow for invariant representation of olfactory information, with significance for downstream processing.

\section{A microfluidic setup for in vivo calcium imaging of larval ORNs}

72 Small size and optical transparency make the larva's olfactory system - like that of $C$. elegans 73 suitable for in vivo multineuronal calcium imaging with precise and flexible microfluidic control of 74 olfactory inputs (Chronis et al., 2007). We developed a microfluidic device for an intact, unanesthetized larva with up to 16 fluid delivery channels, allowing us to image olfactory processing in single animals exposed to a broad input space (Fig 1A-D, Supp Fig 1). Fluid delivery allows for precise control of odorant concentration, timing between stimulus delivery, and stimulus waveform (Andersson et al., 2012). Furthermore, with the microfluidics setup we can record from ensembles of olfactory neurons with single cell resolution while delivering inputs that span odorant types and concentrations. Calcium imaging and genetic labeling allow us to record the activity of any individual ORN alone or the activity of all ORNs simultaneously, by expressing the calcium indicator GCaMP6m (Chen et al., 2013) under the control of either a specific ORN Gal4 driver or the Orco-Gal4 driver, respectively.

\section{Anatomical and functional identification of individual ORNs}

The larva has 21 ORNs located in each bilaterally symmetric dorsal organ ganglion (DOG). The layout of ORN dendrites aids in segmenting and identifying all cells during multineuronal calcium imaging. The 21 ORN sensory dendrites are organized into seven parallel bundles, each containing three sensory dendrites, that project from an ORN soma to the dorsal organ, a perforated dome on the animal's head (Singh and Singh, 1984). When a larva is immobilized in the microfluidic device, four ventral and three dorsal dendritic bundles are easily distinguished (Fig 1E). We mapped individual ORNs to each bundle by expressing RFP in all ORNs and GFP in a selected ORN using a cell specific Gal4 driver (Supp Fig 2). We found that the three ORN dendrites located in each bundle were stereotyped (confirmed in $n \geq 5$ animals for each cell type). Thus, by following the activation of any cell body in the DOG to its corresponding dendritic bundle, its possible identity is narrowed to one of three ORNs. 
98 To further aid in the identification of individual ORNs, we used a set of odorants, termed private

99 odorants, that activate single ORNs at low concentrations. Mathew et al. (2013) assembled a

100 panel of 18 private odorants for each larval ORN by expressing a single functional larval

101 olfactory receptor (Or) in a mutant adult ORN devoid of the endogenous Ors, and recorded its

102 electrical activity in response to olfactory cues. We delivered these private odorants to larvae in

103 our microfluidic setup and found that 18 of the 21 ORNs, in each DOG, are responsive to these

104 odorants: none of the private odorants in the panel activate the Or33a or Or63a ORNs and the

105 Or49a ORN is only activated by a wasp pheromone (Ebrahim et al., 2015). We found that 13 of

106 the private odorants are sufficient to identify all ORNs when examined in conjunction with

107 dendritic bundle location (Fig 1F). Together, the anatomical map and functional responses to

108 this subset of private odorants provides a comprehensive means of identifying and segmenting

109 the ORNs responsive to any olfactory input during multineuronal imaging.

ORN ensemble responses across odorant identities and intensities

112 The panel of 18 private odorants provides a maximally decorrelated set of stimuli that spans the

113 larval olfactory system. To characterize the olfactory representation of these stimuli, we

114 exposed larvae to all 18 private odorants across the concentration range of olfactory sensitivity.

115 We measured the response amplitude of every cell to step stimuli across five orders of

116 magnitude in concentration, from $10^{-8}$ dilution (where all private odorants were at or below

117 threshold of ORN detection) to $10^{-4}$ dilution (where many ORNs had reached saturation). We

118 used five second step pulses interleaved with 20-60 seconds of water, a protocol that allowed

119 us to measure peak responses and allowed for full recovery of neural activity (Supp Fig 3).

121 We verified that all private odorants were highly selective for their target ORNs at low

122 concentrations, with activity expanding to additional ORNs at higher concentrations. For

123 example, 1-pentanol was identified as a private odorant for the Or35a-expressing ORN. At 10-7

124 dilution, 1-pentanol slightly evoked activity specifically in the Or35a-ORN. Higher concentrations

125 of 1-pentanol gradually saturated the Or35a-ORN, while also activating four other ORNs

126 expressing either Or67b, Or85c, O13a, or Or1a (Movie 1). Interestingly, each additional ORN

127 recruited by 1 -pentanol corresponded to a private odorant that is also a long chain alcohol

128 (Mathew et al., 2013). We next examined the ensemble-wide dose-response curves for these

129 additional private alcohol odorants. Low concentrations of each private alcohol specifically

130 activated its target ORN. Higher concentrations reliably activated the Or35a-ORN, Or13a-ORN,

131 Or67b-ORN, and Or85c-ORNs to varying degrees (Supp Fig 4). Furthermore, we performed 
132 the dose-response analysis across the entire ORN ensemble for all 18 private odorants (Fig.

133 2A). We found a similar pattern of overlapping activation for ORNs sharing an odorant with a

134 similar molecular structure. Thus, as odorant concentration increases, a family of molecules with

135 similar structure will cross-activate the subgroup of ORNs that are particularly selective for

136 molecules within the same family.

As in other animals, the olfactory code changes with increasing odorant intensity (Malnic et al., 1999), but with a pattern of ORN recruitment that is correlated with molecular selectivity. To discern this pattern, we used principal component analysis (PCA) of the responses of all ORNs measured against all private odorants across all concentrations. We visualized the data by projecting the ORN activity responses in the space of the first three principal components (PCs) (Fig 2B), which account for $60 \%$ of the variance in the data (Supp Fig 5A). At the lowest concentrations, olfactory representations at or below the detection threshold across odorants were tightly clustered at a central point in the PCA space. At higher concentrations, olfactory representations diverged, increasing distance monotonically from the central point (Fig 2B,

Supp Fig 5B). Interestingly, the trajectory of each odorant tended to follow its own direction in PCA space. This pattern is particularly clear for aliphatic and aromatic odorants. Aliphatic odorants with long carbon chains form trajectories projecting in a similar direction of PCA space, since higher concentrations of these odorants tend to selectively recruit the other ORNs with aliphatic private odorants. The same was true for aromatic odorants and the corresponding group of ORNs with private odorants of this type (Fig 2B). The vectors corresponding to structurally similar molecules were separated by small angles (Fig 2B, Supp Fig 5C). Thus, visualization of ORN responses in PCA space reveals structure in the ensemble representation of odorant identity over a large range of intensities. The population wide response maintains a fixed direction in the representation of each odorant as concentration rises.

\section{Dose-response curves share the same steepness but vary in threshold concentrations} We uncovered additional invariant structure when we analyzed the dose-response relationship of individual odorant-ORN pairs. We found that the subset of all pairs that reached saturation

161 ( $n=21$ of 324 pairs) were well described by a Hill function:

$$
y=y_{\max } \frac{c^{n}}{c^{n}+E C_{50}{ }^{n}},
$$


$E C_{50}$ is the half-maximal effective concentration. The Hill function canonically describes binding affinities in ligand-receptor interactions such as that between odorants and olfactory receptors. Here, we find that the Hill equation describes a common concentration dependent nonlinearity in each dose-response relationship. After normalizing each dose-response curve by $y_{\max }$ and aligning by the $E C_{50}$, all 21 dose-response curves collapsed onto a single Hill function with $n=$ $1.5 \pm 0.1$ (Fig 3A). This common Hill coefficient suggests a similar degree of cooperativity in odorant binding and signal transduction across the ORN repertoire. Assuming the same cooperativity applies to the other odorant-ORN pairs, we estimated the $E C_{50}$ value for all remaining pairs. The complete $E C_{50}$ matrix reveals the distribution of sensitivities across the ORN ensemble to each odorant (Fig 3B).

A simple coding scheme emerges. A common Hill function, with the $E C_{50}$ value as the only free parameter, describes the dose-response relationship for any odorant-ORN interaction. This model, using the complete matrix of estimated $E C_{50}$ values, accounts for $98 \%$ of the variance in the original dataset (Supp Fig 6A). For each odorant, the vector of $E C_{50}$ values (a row in the matrix in Fig 3B) specifies the identity and threshold of each activated ORN with increasing odorant concentration. A corollary of having a unique $E C_{50}$ vector for each odorant is having a unique direction for the trajectory of population responses across concentrations (Fig 2B).

To study structure in the distribution of ORN sensitivities, we applied PCA to the matrix of $\ln \left(1 / E C_{50}\right)$ (see Methods). We found that the first principal component (PC) explains a significant portion of the variance (Supp Fig 6B). We projected the vector of $\ln \left(1 / E C_{50}\right)$ values associated with each private odorant onto this first PC, and found that this projection strongly correlated with aromaticity index (Supp Fig 6C), one of the major quantitative metrics of odorant molecular structure that has been linked to olfactory discrimination across animals (Haddad et al., 2008). This observation explains why the trajectories of aromatic and aliphatic odorant representations point in opposite directions in Fig. 2B.

\section{Power law distribution of ORN ensemble sensitivities}

Next, we examined the properties of the $E C_{50}$ values themselves. We extracted all measured elements from the $E C_{50}$ matrix and constructed a cumulative density function (Fig. $3 \mathrm{C}$ ). The data closely follows a line in the log-log plot, indicating a power law: $P\left(\frac{1}{E C_{50}}\right) \propto\left(\frac{1}{E C_{50}}\right)^{-\lambda-1}, \lambda=$ 0.35 . 
A power law distribution of olfactory sensitivities means that a relative change of concentration will trigger the same mean relative change in the number of activated ORNs, irrespective of odorant type. A power law distribution of olfactory sensitivities, together with a common Hill

202 function, should give rise to ensemble-wide activity that follows a power law relationship with

203 respect to concentration and has an exponent $\lambda$ (See Methods). We confirmed this prediction in 204 our experimental data (Fig 3D). The mean activity of the olfactory ensemble grows with odorant concentration following a power law with an exponent of $0.38 \pm 0.06$, which is close to the 0.35 exponent found from fitting the $E C_{50}$ matrix (Fig $3 \mathbf{C}$ ). Thus, on average, activity expands across the ORN ensemble at the same rate with increasing relative concentration, irrespective of odorant type (as shown in Fig. 2).

\section{ORN-odorant responses share similar temporal characteristics}

211 An additional challenge to olfactory coding of a wide variety of odorant types across

212 concentrations arises from complex temporal dynamics due to physical fluctuations, such as

213 turbulence or convection, in the stimulus itself. To examine how such fluctuations affect ORN

214 responses, we compared the conversion of temporal patterns of olfactory input for different

215 odorant-ORN pairs across odorant intensities. To do this, we used reverse-correlation analysis,

216 subjecting larvae to "white noise" olfactory input by stochastically switching between odorant

217 and water delivery and seeking the temporal filter that best maps olfactory inputs into calcium

218 dynamics (Geffen et al., 2009; Kato et al., 2014). We found that random olfactory input could

219 evoke fluctuating calcium activity in an ORN, and repeated presentation of the same input

220 pattern would evoke consistent responses from trial to trial (Supp Fig 7A). The systematic

221 conversion of the stimulus to response waveform is well characterized by a linear-nonlinear (LN)

222 model. A linear transfer function estimates the relative weight of each time point in stimulus

223 history to determine the time-varying response amplitude (Supp Fig 7B). The convolution of the

224 linear transfer function with stimulus history is then passed through a static nonlinearity to

225 correct for saturation (Supp Fig 7C). We verified the LN model by predicting the response to a

226 novel random input using a filter calculated from different random inputs (Supp Fig 7D).

228 We measured the linear transfer function for 3-octanol, the private odorant for the Or85c-ORN, 229 across the concentration range used to characterize the $E C_{50}$ matrix. At the lowest 230 concentrations of 3-octanol, a filter describing ORN activity only emerges for the Or85c-ORN 231 (Fig 4A). At higher concentrations, filters begin to emerge for additional ORNs. These filters for 
each ORN, when normalized for response amplitude, were virtually identical in their temporal response profiles as single lobed functions with similar peak and decay times (Fig 4B, Supp

234 Fig 8B-C). The shapes of the filters for different odorants activating the same ORN are also 235 virtually indistinguishable, on the order of $\sim 100 \mathrm{~ms}$ (Fig 4C, Supp Fig 8A-C). This result is

236 constrained by the calcium indicator, which has a time constant associated with calcium binding 237 to GCaMP6m, making it difficult to resolve differences in ORN temporal filters on a shorter time 238 scale. Nonetheless, recent electrophysiological measurements of odorant-evoked activity in the 239 ORNs of adult Drosophila, under the same LN model, also report remarkable similarity in the temporal pattern of filters across odorant-receptor pairs, within 10-20 ms (Martelli et al., 2013).

A common temporal filter across ORNs could simplify the olfactory code in an environment with fluctuating odorant concentrations. A constant filter in conjunction with uniform scaling of ORN activity over concentration could allow the ensemble of responsive neurons to maintain the same relative amplitudes of activation over time. These relative amplitudes would be correlated with the ORN ensemble $E C_{50}$ values for any odorant, regardless of whether an animal is in a static or fluctuating odorant environment.

\section{Discussion}

250 Previous efforts at a systems-level characterization of ORNs necessarily focused analysis on particular cell types, odorants, or odorant concentrations (Hallem and Carlson, 2006; Mathew et al., 2013; Nagel and Wilson, 2013; Martelli et al., 2013; Asahina et al., 2009). The small size of the Drosophila larva, combined with multineuronal imaging and new microfluidic tools, has allowed us to characterize the responses of a complete ORN ensemble to a panel of odorant types and concentrations that spans the selectivity of olfactory sensory neurons. This broad characterization has uncovered regular patterns in the response of individual ORNs and of the ORN ensemble. First, each ORN response to odorants exhibits the same activation function

258 shape with variant sensitivity levels. Furthermore, consistent temporal filters that convert 259 different stimulus waveforms into ORN calcium activity patterns will make the relative activities 260 of different ORNs robust despite fluctuating inputs. Second, the ORN ensemble across all 261 tested odorants exhibits a constant rate of increase in activity with increasing concentration. 262 Underlying this effect, we have identified a power-law distribution in the sensitivities of odorant263 ORN interactions. The power law distribution may allow downstream circuits to estimate the 264 relative concentrations of any odorant by the relative extent of ORN activity using the same 265 quantitative relationship for any odorant. Invariant quantitative patterns in single and ensemble- 
level ORN activities could allow shared mechanisms to extract olfactory features, even in fluctuating environments, across olfactory space.

Different neurons are required to sense odorants in different regimes of odorant concentration needed for long-range chemotaxis in the Drosophila larva (Asahina et al., 2009). Encoding a broad concentration range requires a distribution of ORNs with varying sensitivities. Our analysis reveals that olfactory sensitivities follow an invariant statistical distribution across odorants and ORN types. This power law distribution implies a fixed ratio between the relative change in ORN ensemble activity for a fixed change in odorant concentration. Detection of relative change in stimulus intensities has been observed in psychophysical studies of diverse sensory modalities. A notable example is Stevens's Law in human psychophysics, where response magnitudes have been shown to scale with the logarithm of stimulus intensities across sensory modalities including olfaction (Stevens, 1957). Our results reveal that a phenomenon analogous to Stevens's Law can be attributed to the olfactory sensory periphery itself, a direct outcome of the statistical distribution of response sensitivities across an ensemble.

A combinatorial olfactory code will arise from a distribution of ORN sensitivities to different odorant molecules. Changes in concentration necessarily lead to changes in the combinatorial code, but with correlated changes depending on the odorant. The basis of this correlation is a unique vector of sensitivities across ORNs for each odorant. This constraint allows each odorant's identity to be coded in a concentration-independent manner as a direction in an olfactory coding space. It has been suggested that extracting relative glomerulus activity across odorant concentrations may allow the concentration-invariant coding of different odorant types (Wachowiak et al., 2002; Cleland et al., 2007). For animals that sniff, the change in concentration through inhalation generates a reliable temporal sequence of ORN activity that could represent the vector of ORN sensitivity (Smear et al., 2011). As we have found in Drosophila, a common activation function and temporal filter - which may arise from stereotyped receptor cooperativities and shared transduction dynamics among ORNs - would

294 facilitate the decoding that takes place by such mechanisms to extract concentration invariant 295 representations of odorant identity.

297 To our knowledge, a power law distribution of olfactory sensitivities has not yet been described 298 in any animal. One possibility for the power law in olfactory sensitivity is to match the 299 distributions of odorant concentrations found in natural olfactory environments. Natural odors 
are mixed by convection and turbulence, physical processes that are rich in power law dynamics (Catrakis and Dimotakis, 1996). Power laws appear in the statistics of other natural stimuli as well. Natural visual scenes exhibit a power law relationship between spectral power and spatial frequency (Field, 1987; Simoncelli and Olshausen, 2001). The loudness of natural sounds across frequencies are distributed by power laws (Theunissen and Elie, 2014). Sensory systems, in general, may adapt the statistical distribution of their sensitivities to their natural environments.

Another possibility is that the observed power law distribution represents an optimization of the olfactory code. It has recently been proposed that the olfactory code maximizes informational entropy (Stevens, 2015). Given the constraint of fixed mean firing rates among ORNs, this model leads to an exponential distribution of ORN firing rates evoked by odorants measured in the adult Drosophila antenna (Stevens, 2016). Interestingly, another prediction of this optimization is that the overall activity of the olfactory ensemble should increase as a power law of odor concentration, as we have also experimentally observed in the Drosophila larva and, in our case, connected to the statistical distribution of olfactory sensitivities across ORNs. a power law distribution in ORN sensitivities. Lancet et al., 1993 proposed a molecular recognition system in which a receptor has multiple selective binding sites. Each binding site contributes in a combinatorial manner to the binding strength between a receptor and molecule. This simple quantitative model generates a power law sensitivity distribution for receptors with random sets of binding sites. The statistics of an olfactory code using such a molecular recognition system would be robust to expansion of the ORN periphery, as occurs with Drosophila in which the adult has nearly triple the number of receptor types as that found in the larva. Furthermore, a conserved statistical structure in the olfactory code would allow downstream circuitry to employ similar decoding mechanisms across an animal's lifetime.

\section{Acknowledgment}

329 The authors would like to thank the valuable suggestions from Benjamin de Bivort, Dmitri 330 Chklovskii, Cengiz Pehlevan, Marta Zlatic, Betty Hong, and Yuhai Tu. This work was performed 331 in part at the Harvard Center for Nanoscale Systems, a member of the National Nanotechnology 332 Infrastructure Network (NNIN), which is supported by the National Science Foundation under 333 NSF award no. ECS-0335765. This work was also supported by a National Science Foundation 
bioRxiv preprint doi: https://doi.org/10.1101/208538; this version posted October 25, 2017. The copyright holder for this preprint (which was not certified by peer review) is the author/funder, who has granted bioRxiv a license to display the preprint in perpetuity. It is made available under aCC-BY-NC-ND 4.0 International license.

334 Graduate Research Fellowship (DGE1144152), NSF Brain Initiative grant (NSF-IOS-1556388), 335 and grants from the NIH (8DP1GM105383, P01GM103770, F31DC015704). YH acknowledges 336 support from the Swartz Program in Theoretical Neuroscience at Harvard.

337 
A

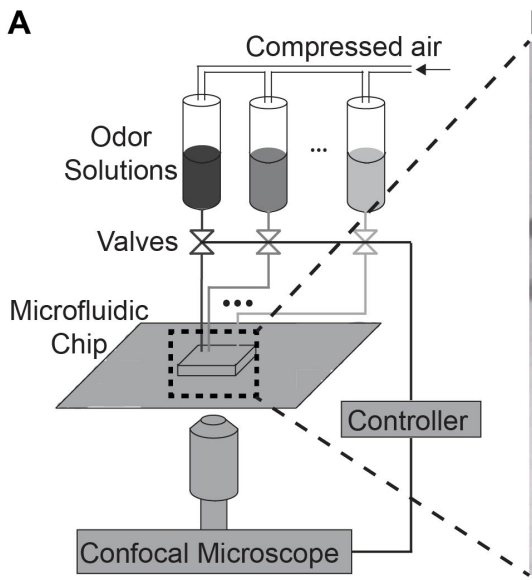

B

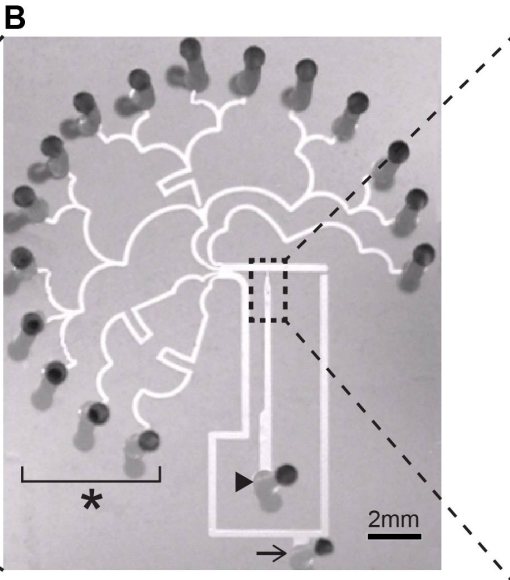

C

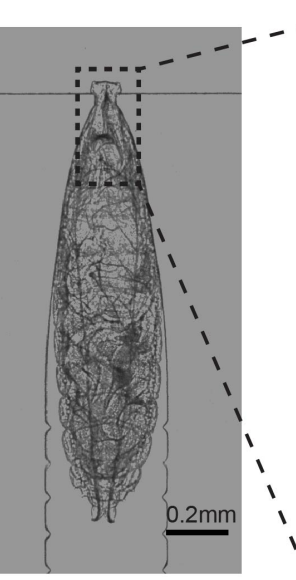

D

E

Ventral View
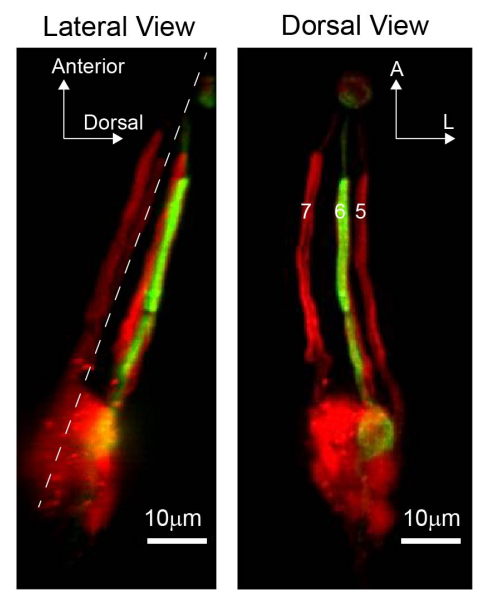

$\mathbf{F}$
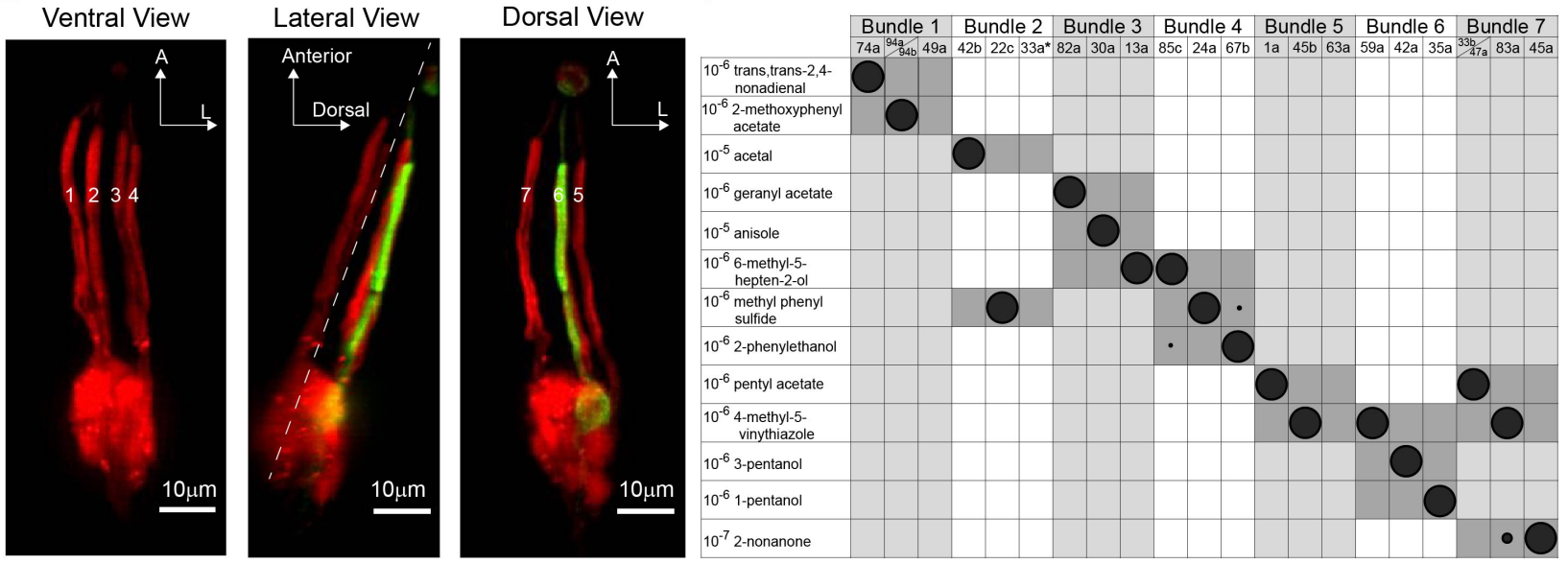

Figure 1. Anatomical and functional identification of individual ORNs within the ORN

population. A. Schematic of the setup for confocal imaging of a larva in a microfluidic device during odorant delivery.

B. 16 channel microfluidic device ( ${ }^{*}$ indicates stimulus delivery channels, arrowhead marks larva loading inlet, arrow marks fluid outlet). C and D. Zoomed-in view of an immobilized larva in the loading channel. Red indicates RFP labeling of ORN dendrites and cell bodies. E. Larval ORN sensory dendrites are organized into seven parallel bundles (numbered). All ORNs shown in red, Or35-ORN shown in green, using Or35a>GFP; Orco>RFP genotype. Dashed line in lateral view marks separation between ventral and dorsal bundles. F. Each of 13 odorants at low concentrations primarily activates only one ORN within each bundle. Size of shaded circles indicates normalized neural activity $(\Delta F / F)$ of the specified ORN to an odorant. * indicates that location of Or33a-ORN was inferred from vacancy in bundle 2 (Supp Fig 2). 
bioRxiv preprint doi: https://doi.org/10.1101/208538; this version posted October 25, 2017. The copyright holder for this preprint (which was not certified by peer review) is the author/funder, who has granted bioRxiv a license to display the preprint in perpetuity. It is made available under aCC-BY-NC-ND 4.0 International license.

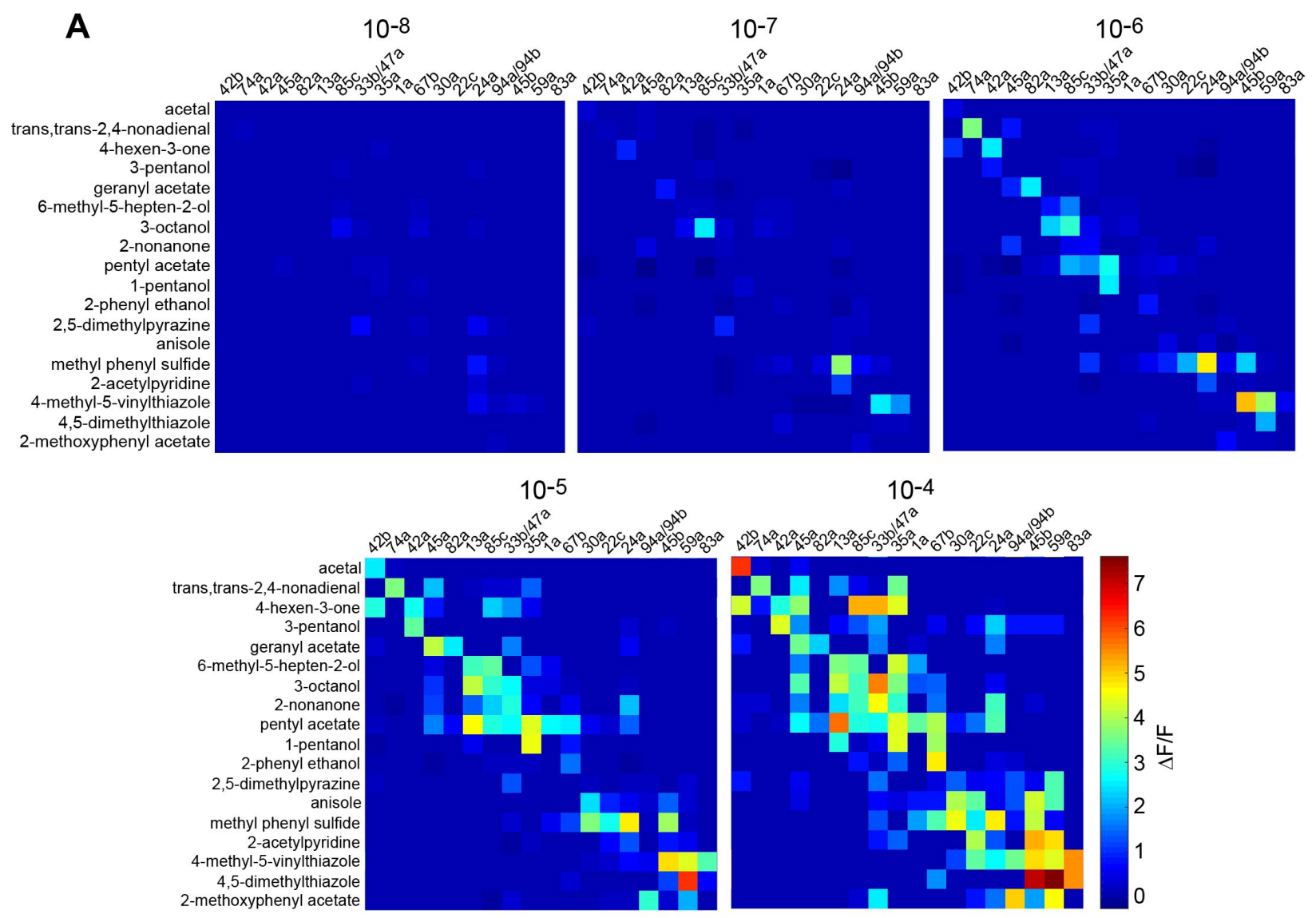

\section{B}
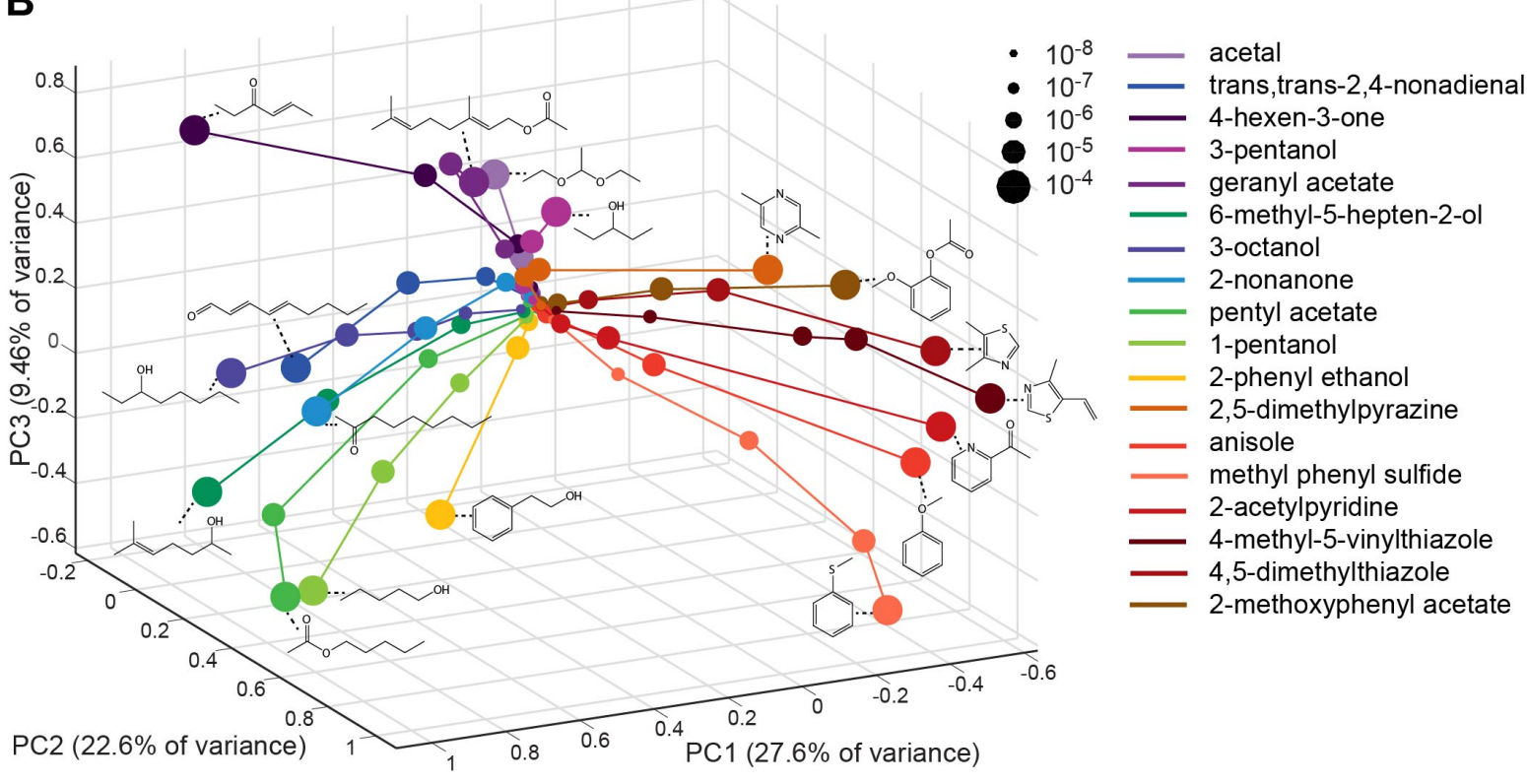

Figure 2. ORN population responses to different odorants and concentrations. A.

Averaged peak responses of 18 ORNs to a panel of 18 odorants, each delivered at five concentrations ( $n \geq 5$ for each odorant type and concentration; odorant pulse $=5 \mathrm{~s}$ ). B. ORN 
357 population responses visualized in PCA space. Each dot represents the projection of ORN

358 population activity onto the first three principal components. Size and color of dots correspond

359 to odorant concentration and type, respectively. Dots from the same odorant are linked and the

360 molecular structure of the odorant is shown adjacent to each trajectory. Aromatic versus

361 aliphatic odorants cluster in separate regions of PCA space. 


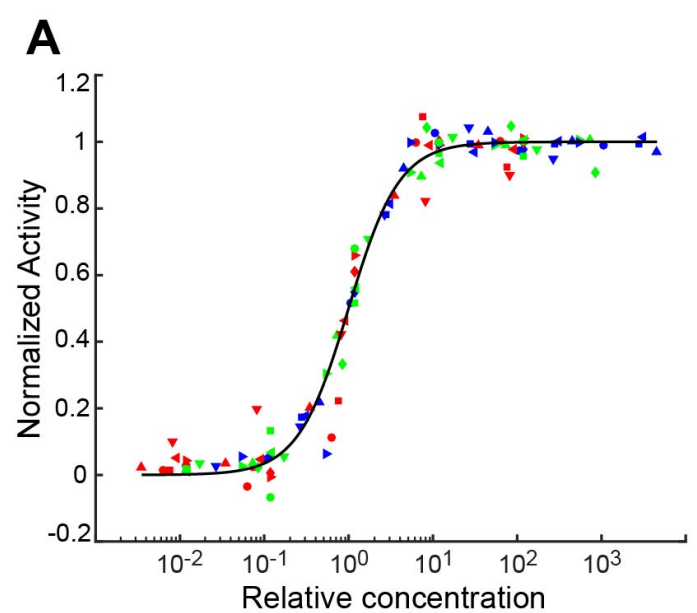

C

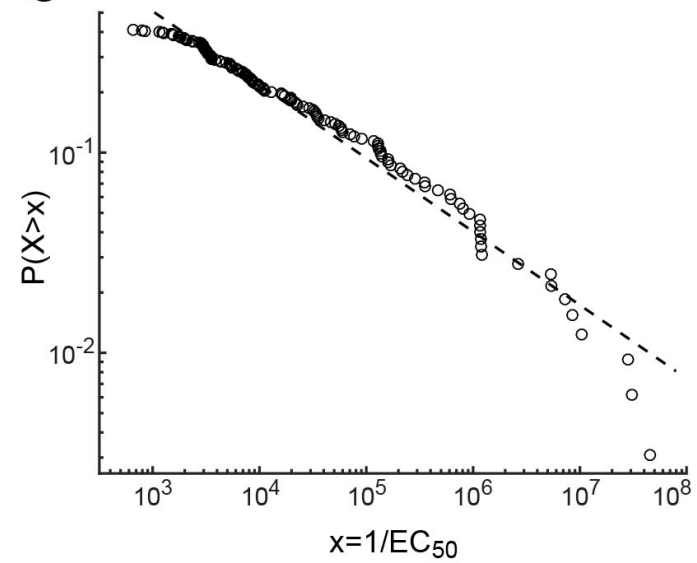

B

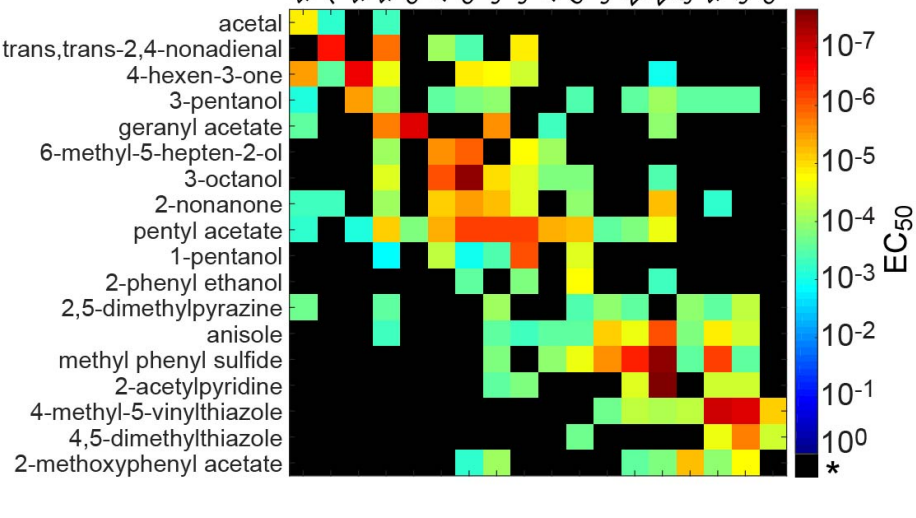

D

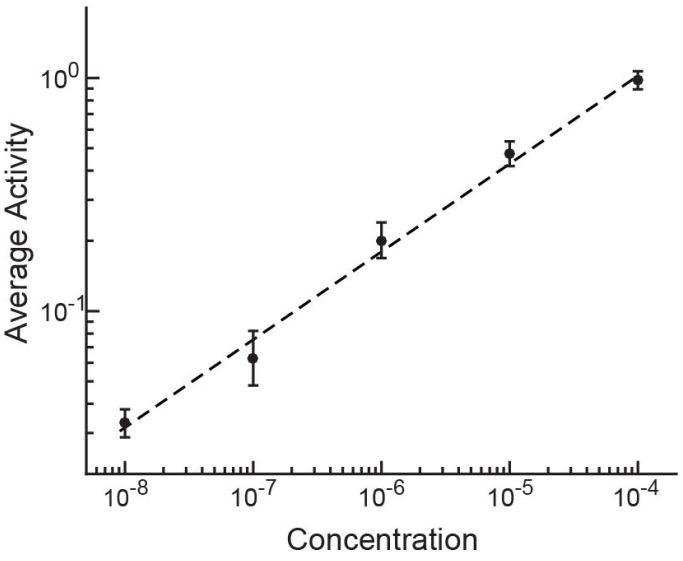

Figure 3. Scaling laws for individual and ensemble ORN activity. A. Normalized ORN responses for various odorant-ORN pairs across relative odorant concentration (actual concentration divided by $E C_{50}$ ). Individual curves for plotted odorant-ORN pairs collapse onto a single curve described by a Hill equation with a shared Hill coefficient of 1.45. Black line indicates the fitted Hill equation, different colored and shaped points represent data from unique odorant-ORN pairs. B. Matrix of $E C_{50}$ values fit to dose-response data from each odorant-ORN pair ( ${ }^{*}$ for black squares indicates that odorant-ORN pair had no response within the tested concentration range). C. Log-log plot of the cumulative distribution function of $1 / E C_{50}$ values. The dashed line is a linear fit to the data and has a slope of -0.35. D. Log-log plot of average neuron activity across all odorant-ORN pairs for each concentration. The error bars represent the standard error. Least-squares fit line has a slope of $0.38 \pm 0.06\left(R^{2}=0.99\right)$. 

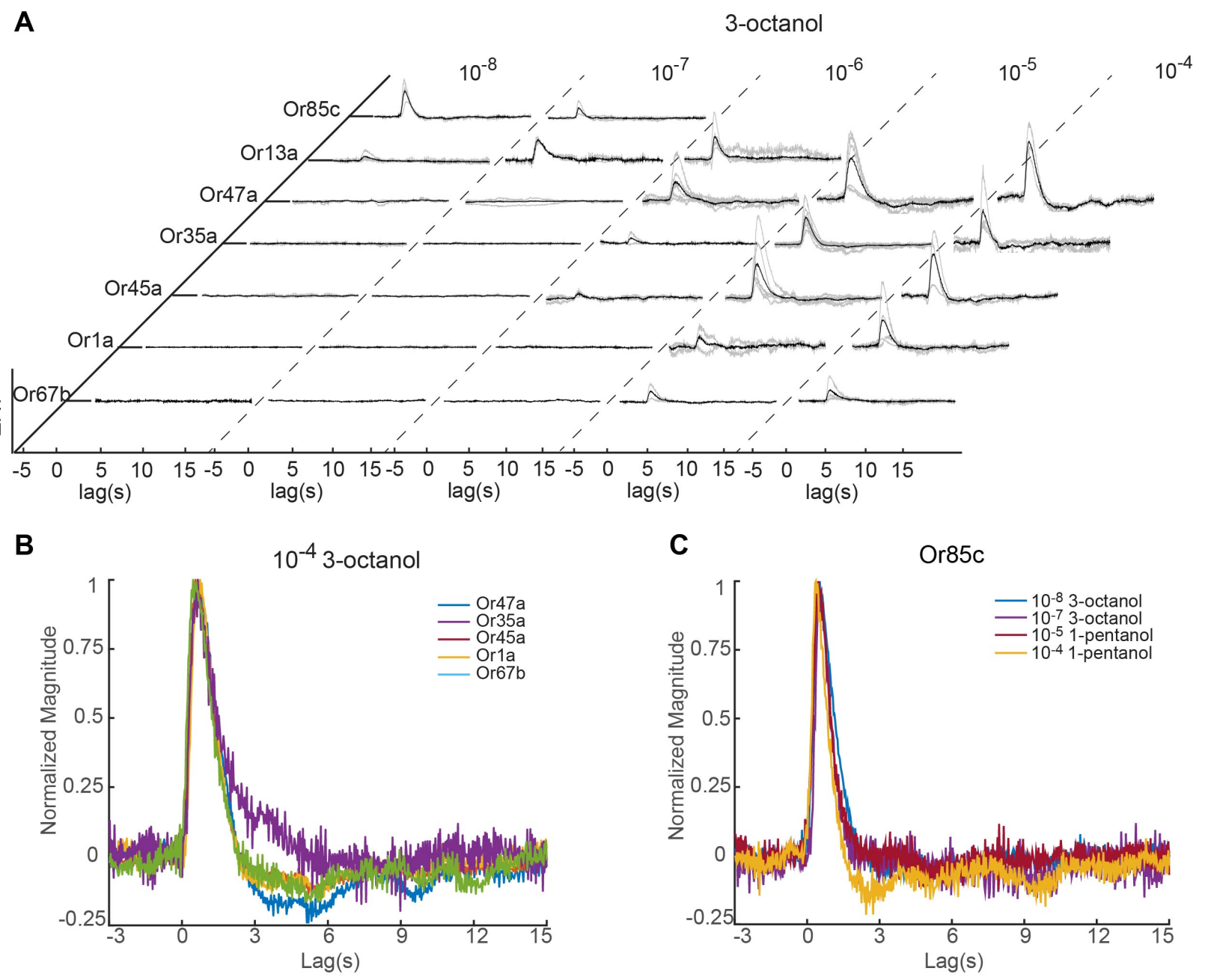

C

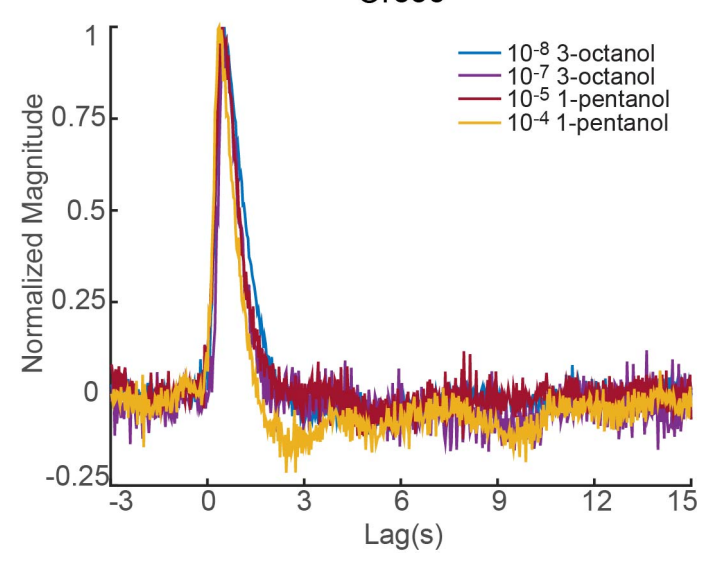

Figure 4. Temporal filters of ORN response. A. Linear filters of 7 ORNs responding to 3octanol across five concentrations. Black curve indicates the averaged filter from data across

377 multiple animals (individual filters shown in gray). B and C. Comparison of filter waveforms for 378 the same odorant $\left(10^{-4}\right.$ dilution of 3-octanol) activating different ORNs (B), and the same ORN 379 (Or85c-ORN) responding to different odorants and concentrations (C). All filters were normalized by their peak amplitude. 


\section{References:}

1. Malnic, B., Hirono, J., Sato, T. \& Buck, L. B. Combinatorial receptor codes for odors. Cell, 96(5), 713-723 (1999).

2. Buck, L., \& Axel, R. A novel multigene family may encode odorant receptors: a molecular basis for odor recognition. Cell, 65(1), 175-187 (1991).

3. Friedrich, R. W. \& Korsching, S. I. Combinatorial and chemotopic odorant coding in the zebrafish olfactory bulb visualized by optical imaging. Neuron, 18(5), 737-752 (1997).

4. Hallem, E. A. \& Carlson, J. R. Coding of odors by a receptor repertoire. Cell, 125(1), 143-160 (2006).

5. Kreher, S. A, Kwon, J. Y. \& Carlson, J. R. The molecular basis of odor coding in the Drosophila larva. Neuron, 46(3), 445-56 (2005).

6. Nara, K., Saraiva, L. R., Ye, X. \& Buck, L. B. A large-scale analysis of odor coding in the olfactory epithelium. Journal of Neuroscience, 31(25), 9179-9191 (2011).

7. Kajiya, K., Inaki, K., Tanaka, M., Haga, T., Kataoka, H. \& Touhara, K. Molecular bases of odor discrimination: reconstitution of olfactory receptors that recognize overlapping sets of odorants. Journal of Neuroscience, 21(16), 6018-6025 (2001). imaging reveals an odor-evoked map of activity in the fly brain. Cell, 112(2), 271-282 (2003).

9. Friedrich, R. W. \& Laurent, G. Dynamic optimization of odor representations by slow temporal patterning of mitral cell activity. Science, 291(5505), 889-894 (2001).

10. Laurent, G., Stopfer, M., Friedrich, R. W., Rabinovich, M. I., Volkovskii, A. \& Abarbanel, H. D. Odor encoding as an active, dynamical process: experiments, computation, and theory. Annual Review of Neuroscience, 24(1), 263-297 (2001).

11. Junek, S., Kludt, E., Wolf, F. \& Schild, D. Olfactory coding with patterns of response latencies. Neuron, 67(5), 872-884 (2010).

12. Smear, M., Shusterman, R., O'Connor, R., Bozza, T. \& Rinberg, D. Perception of sniff phase in mouse olfaction. Nature, 479(7373), 397-400 (2011).

13. Nagel, K. I. \& Wilson, R. I. Biophysical mechanisms underlying olfactory receptor neuron dynamics. Nature Neuroscience, 14(2), 208-216 (2011).

14. Martelli, C., Carlson, J. R. \& Emonet, T. Intensity invariant dynamics and odor-specific latencies in olfactory receptor neuron response. Journal of Neuroscience, 33(15), 62856297 (2013). 
15. Wachowiak, M., Cohen, L. B. \& Zochowski, M. R. Distributed and concentration-invariant spatial representations of odorants by receptor neuron input to the turtle olfactory bulb. Journal of Neurophysiology, 87(2), 1035-1045 (2002).

16. Cleland, T. A., Johnson, B. A., Leon, M., \& Linster, C. Relational representation in the olfactory system. Proceedings of the National Academy of Sciences, 104(6), 1953-1958 (2007).

17. Stevens, C. F. A statistical property of fly odor responses is conserved across odors. Proceedings of the National Academy of Sciences, 113(24), 6737-6742 (2016).

18. Vosshall, L. B. \& Stocker, R. F. Molecular architecture of smell and taste in Drosophila. Annu. Rev. Neurosci., 30, 505-533 (2007).

19. Su, C. Y., Menuz, K. \& Carlson, J. R. Olfactory perception: receptors, cells, and circuits. Cell, 139(1), 45-59 (2009).

20. Chronis, N., Zimmer, M. \& Bargmann, C. I. Microfluidics for in vivo imaging of neuronal and behavioral activity in Caenorhabditis elegans. Nature methods, 4(9), 727-731 (2007).

21. Andersson, M. N., Schlyter, F., Hill, S. R., \& Dekker, T. What reaches the antenna? How to calibrate odor flux and ligand-receptor affinities. Chemical senses, 37(5), 403-420 (2012).

22. Chen, T. W., Wardill, T. J., Sun, Y., Pulver, S. R., Renninger, S. L., Baohan, A., ... \& Looger, L. L. Ultrasensitive fluorescent proteins for imaging neuronal activity. Nature, 499(7458), 295-300 (2013).

23. Singh, R. N., \& Singh, K. Fine structure of the sensory organs of Drosophila melanogaster Meigen larva (Diptera: Drosophilidae). International Journal of Insect Morphology and Embryology, 13(4), 255-273 (1984).

24. Mathew, D., Martelli, C., Kelley-Swift, E., Brusalis, C., Gershow, M., Samuel, A. D., Emonet, T. \& Carlson, J. R. Functional diversity among sensory receptors in a Drosophila olfactory circuit. Proceedings of the National Academy of Sciences, 110(23), E2134-E2143 (2013).

25. Ebrahim, S. A., Dweck, H. K., Stökl, J., Hofferberth, J. E., Trona, F., Weniger, K., ... \& Hansson, B. S. Drosophila avoids parasitoids by sensing their semiochemicals via a dedicated olfactory circuit. PLoS Biology, 13(12), e1002318 (2015).

26. Haddad, R., Khan, R., Takahashi, Y. K., Mori, K., Harel, D. \& Sobel, N. A metric for odorant comparison. Nature Methods, 5(5), 425-429 (2008).

27. Geffen, M. N., Broome, B. M., Laurent, G. \& Meister, M. Neural Encoding of Rapidly Fluctuating Odors. Neuron, 61(4), 570-586 (2009). 
28. Kato, S., Xu, Y., Cho, C. E., Abbott, L. F. \& Bargmann, C. I. Temporal responses of C. elegans chemosensory neurons are preserved in behavioral dynamics. Neuron, 81(3), 616-628 (2014).

29. Asahina, K., Louis, M., Piccinotti, S. \& Vosshall, L. B. A circuit supporting concentrationinvariant odor perception in Drosophila. Journal of Biology, 8(1), 9 (2009).

30. Stevens, S. S. On the psychophysical law. Psychological review, 64(3), 153 (1957).

31. Catrakis, H. J. \& Dimotakis, P. E. Scale distributions and fractal dimensions in turbulence. Physical Review Letters, 77(18), 3795 (1996).

32. Field, D. J. Relations between the statistics of natural images and the response properties of cortical cells. Journal of the Optical Society of America A, 4(12), 2379-2394 (1987).

33. Simoncelli, E. \& Olshausen, B. Natural Image Statistics and Neural Representation. Annual Review of Neuroscience, 24, 1193-1216 (2001).

34. Theunissen, F. E. \& Elie, J. E. Neural processing of natural sounds. Nature Reviews Neuroscience, 15(6), 355-366 (2014).

35. Stevens, C. F. What the fly's nose tells the fly's brain. Proceedings of the National Academy of Sciences, 112(30), 9460-9465 (2015). biological receptor repertoires: significance to the olfactory system. Proceedings of the National Academy of Sciences, 90(8), 3715-3719 (1993). 


\section{Methods}

\section{Fly stocks}

470 Flies were reared at $22^{\circ} \mathrm{C}$ under a $12: 12$ hour light/dark cycle in vials containing conventional

471 yeast agar medium. Adult flies were transferred to a larvae collection cage (Genesee Scientific)

472 containing a grape juice agar plate and a dime-sized amount of fresh yeast paste. Flies could

473 lay eggs on the grape juice agar plate for two days and then the plate was removed for

474 collection of first instar larvae. The following fly lines were used in this study: UAS-

475 mCherry.NLS; UAS-GCaMP6m, UAS-mCD8::GFP; Orco::RFP, Orco-Gal4 (BL23292), Or1a-

476 Gal4 (BL9949), Or13a-Gal4 (BL9945), Or22c-Gal4 (BL9953), Or24a-Gal4 (BL9958), Or30a-

477 Gal4 (BL9960), Or33b-Gal4 (BL9963), Or35a-Gal4 (BL9968), Or42a-Gal4 (BL9970), Or42b-

478 Gal4 (BL9971), Or45a-Gal4 (BL9976), Or45b-Gal4 (BL9977), Or47a-Gal4 (BL9982), Or49a-

479 Gal4/Cyo; Dr/TM3 (gift from John Carlson lab), Or59a-Gal4 (BL9990), Or63a-Gal4 (BL9992),

480 Or67b-Gal4 (BL9995), Or74a-Gal4 (BL23123), Or82a-Gal4 (BL23125), Or83a-Gal4 (BL23128),

Or85c-Gal4 (BL23913), Or94b-Gal4 (BL23916).

\section{Microfluidic device design, fabrication, and calibration}

484 Odorant stimuli were delivered using a microfluidic device (Fig 1A) designed with a $300 \mu \mathrm{m}$

wide and $70 \mu \mathrm{m}$ high larva loading channel. The channel tapered to a width of $60 \mu \mathrm{m}$ in order to immobilize the larva. The tapered end was positioned perpendicular to a stimulus delivery channel to allow for odorant flow past larval ORNs. The device was designed with a "shiftingflow strategy", similar to that described in Chronis et al, 2007. The 16-channel device included two control channels located at the periphery, 13 odorant channels in the middle, and one water channel to remove odorant residue. Each channel was of equal length to ensure equal resistance. During an experiment, a combination of three channels was always open: the water channel, one of the 13 odorant delivery channels, and one of the control channels. The 13 odorant channels could be sequentially opened to deliver any odorant. Switching between the two control channels directed either water or an odorant to flow past the larva's ORNs, as demonstrated in Supp Fig 1.

Fluorescein dye was used to measure the switching time between water and odorants as well 498 as to verify the spatial odorant profile in the device during stimulus delivery. Our standard air 499 pressure for stimulus delivery was $6 \mathrm{psi}$, which led to a flow rate of $0.5 \mathrm{~mL} / \mathrm{min}$ in the microfluidic 500 device. With these conditions, the switching time between water and odorant was $\sim 20 \mathrm{~ms}$ 
502 The microfluidic device pattern was designed using AutoCAD. The design pattern was then

503 transferred onto a silicon wafer using photolithography. The wafer was used to fabricate

504 microfluidic devices using polydimethylsiloxane (PDMS) and following the standard soft

505 lithography approach (Anderson et al, 2000). The resulting PDMS molds were cut and bonded

506 to glass cover slips. Each microfluidic device was used for only a single panel of odorants in

507 order to prevent contamination.

\section{Odorant delivery setup}

510 Odorants were obtained from Sigma-Aldrich, diluted in deionized (DI) water (Millipore) and

511 stored for no more than 2 days. To prevent contamination, each odorant concentration was

512 stored in a separate glass bottle and delivered through its own syringe and tubing set. Panels of

513 odorants were delivered using a 16-channel pinch valve perfusion system (AutoMate Scientific,

514 Inc.). Each syringe and tubing set contained a $30 \mathrm{~mL}$ luer lock glass syringe (VWR) connected

515 to Tygon FEP-lined tubing (Cole-Parmer), which in turn was connected to silicone tubing

516 (AutoMate Scientific. Inc.). The silicone tubing was placed through the pinch valve region of the

517 perfusion system as its flexibility could allow for the passage or blockage of fluid flow to the

518 microfluidics device. The silicone tubing was then connected to PTFE tubing (Cole-Parmer),

519 which was then inserted into the microfluidic device. A microcontroller and custom written

520 Matlab code were used to control the on/off sequence of the valves and to synchronize valve

521 control with the onset of recording in the imaging software (NIS Elements).

523 During the entire recording, the larva experienced continuous fluid flow, with a flow rate of

$5240.5 \mathrm{~mL} / \mathrm{min}$ or $0.2 \mathrm{~m} / \mathrm{s}$. In the dose-response experiments, the stimuli sequences consisted of five

525 seconds of odorant pulses followed by a washout period using water. The duration of odorant

526 pulses was chosen such that ORN responses reached maximum amplitude. The washout time

527 was adjusted to allow for ORN recovery back to baseline activity levels, and thus ensured that

528 measurements of ORN responses were independent of stimulus sequence (Supp Fig 3 and

529 Movie 1). For the white noise experiments, a 1024-step m-sequence of odorant stimulus and

530 water was delivered with a time step of $0.2 \mathrm{~s}$ (Movie 2).

\section{Calcium imaging}

533 A first instar larva was loaded into a microfluidic device using a $1 \mathrm{~mL}$ syringe filled with $0.1 \%$

534 triton-water solution. Using the syringe, a larva was pushed towards the end of the channel,

535 where the $60 \mu \mathrm{m}$ wide opening mechanically trapped further larval movement. Each larva was 
536 positioned such that its dorsal organ (nose) was exposed to the stimulus delivery channel and

537 its dorsal side (where ORN cell bodies are located) was closest to the objective. Larvae were

538 imaged using an inverted Nikon Ti-e spinning disk confocal microscope with a 60X water

539 immersion objective (NA 1.2). A charged-coupled device (CCD) microscope camera (Andor

540 iXon EMCCD) captured images at 30 frames/sec. ORN cell bodies were recorded by scanning

541 the entire volume ( 20 slices with a step size of $1.5 \mu \mathrm{m}$ ) of the dorsal organ ganglion (Movie 1),

542 while ORN axon terminals were recorded from a single slice of the antennal lobe (Movie 2).

543 Dose-response experiments (data shown in Fig 1-2, Supp Fig 3-4 and Movie 1) were

544 performed using larvae of the Orco $>$ GCaMP6m, Orco>mCherry.NLS genotype and recording

545 from ORN cell bodies. White noise experiments (data shown in Fig 4, Supp Fig 7-8 and Movie

546 2) were performed using larvae expressing GCaMP6m in a single ORN (e.g. Or42a>GCaMP6m

547 used in Supp Fig 7) and recording from ORN axon terminals.

Dose response analysis

550 Custom code written in ImageJ was used to track and identify each ORN as well as its responses to odorant stimuli. Slight movement artifacts were corrected by aligning frames using mCherry NLS labeling of ORN cell bodies and the ImageJ TurboReg plugin (Thevenaz et al, 1998). Each ORN activated in response to an odorant stimulus was visually identified using both

554 the anatomical location of its dendritic bundle and the functional map of cognate odorant to 555 ORN activation (Fig 1 E, F, Supp Fig 2). ORN identification was performed independently by 556 two experimenters to ensure accuracy. Changes in fluorescence were then quantified as $\left(F_{\text {peak }}-F_{0}\right) / F_{0}$, where $F_{0}$ was the average ORN intensity sampled from the frames immediately preceding odorant delivery and $F_{\text {peak }}$ was the highest intensity in ORN fluorescence during odorant delivery. Each odorant stimulus was repeated with at least 5 trials. The raw response data is summarized in Supp Table 1.

The heatmap in Fig 2A was generated by directly averaging the peak responses across trials. Simulated annealing was used to optimize the order of ORNs and odorants presented in this heatmap, such that it minimized a loss function in which cost increased linearly with the distance that activated odorant-ORN pairs were from the matrix diagonal. The response data was normalized by the highest response level within each trial, averaged across trials, and then Zscored prior to performing PCA. The distance and direction of vectors shown in Supp Fig 5 were calculated for each data point in Fig 2B using the standard formulae for cartesian to polar coordinate transformation. 


\section{Dose-response curve fitting}

572 A Hill equation with a unique set of parameter values was fit to the dose-response curve for

573 each odorant-ORN pair in our data set. The general form of the Hill equation is as follows:

$$
y=y_{\max } \frac{c^{n}}{c^{n}+E C_{50}{ }^{n}}
$$

where $y_{\max }$ is the maximum ORN response level across concentrations, $c$ is the odorant concentration, $\mathrm{EC}_{50}$ is the half-maximal effective concentration, and $n$ is the Hill coefficient. In the calcium imaging experiment, the maximum fluorescence intensity $y_{\max }$ could be affected by the detailed experimental settings and it is differently fitted for the curve of each odorantORN pair. Here, the absolute value of $y_{\max }$ is not considered a coding feature, so in the following analysis, we normalized the responses using $y_{\max }$.

There were 21 odorant-ORN pairs saturated within the concentration range we studied. We started by fitting these 21 curves using the Hill equation. We normalized the responses using the $y_{\max }$ for each odorant-ORN pair and shifted the x-axis using its $E C_{50}$. A scatter plot of the normalized and shifted dose-response data for the 21 odorant-ORN pairs is shown in Fig 3A.

Next, we used a Hill equation function with $y_{\max }=1$ and $E C_{50}=0$ to fit all 105 data points from the 21 different odorant-ORN pairs. The resulting equation had a Hill coefficient $\mathrm{n}=1.45$, with $\mathrm{R}^{2}>0.99$. Next, we applied this Hill coefficient to fit odorant-ORN data pairs that did not saturate in the concentration range we had tested. There were 19 additional odorant-ORN pairs that were close to saturation and we could therefore estimate their $y_{\max }$ and $E C_{50}$ values well.

After fitting the 21 odorant-ORN pairs that had saturated as well as the 19 that were close to saturation, we had at least one parametrized Hill equation for each odorant. To fit the remaining odorant-ORN pairs that were not close to saturation within our tested concentration range, we first assumed that each odorant had approximately the same $y_{\max }$ for each odorant (this was calculated by averaging the $y_{\max }$ of all ORNs that shared an odorant with a parameterized logistic curve). Given the known $y_{\max }$ for each odorant and the fixed Hill coefficient, we could estimate the $E C_{50}$ for the remaining 100 weakly responding odorant-ORN pairs. indicate that the corresponding ORN showed no activity within the tested concentration range; 
603 we were unable to fit an $E C_{50}$ value for these odorant-ORN pairs. We used the Hill equation and

604 fitted parameters for each odorant-ORN pair to generate the activity response data and found

605 that it was similar to the actual data (Supp Fig 6A).

\section{Analysis of the $E C_{50}$ Matrix}

608 To perform PCA on the $E C_{50}$ matrix, we first transformed the values to the $-\ln \left(E C_{50}\right)$, such that 609 odorant-ORN pairs with a high sensitivity (small $E C_{50}$ ) were now represented by large values 610 and those that were less sensitive (large $E C_{50}$ ) had small values. The remaining pairs that that 611 did not have an $E C_{50}$ value (the missing data, represented by black squares in Fig 3B),

612 represent pairs with a much lower sensitivity and were set to zero. Supp Fig 6B shows the 613 percentage of variance explained by each principal component (PC) once PCA was performed 614 on the $-\ln \left(E C_{50}\right)$ matrix. In comparison to a shuffled matrix (in which each row is randomly 615 permuted), we found that only the first PC was significantly different $(p<0.0001$ for 1000 616 instances of shuffled data).

618 We compared 32 descriptors of molecular structure from the E-Dragon software, which were 619 found in Haddad et al., 2008 to be relevant for olfactory coding across animals. We found that 620 one metric, aromaticity index of a molecule, had the highest correlation with the first PC of the $621 E C_{50}$ matrix with a coefficient of 0.8 (Supp Fig 6C). We fit the power law distribution using code from (Clauset et al., 2009). The resulting fitting 624 index of 0.22 (large values mean better fit to the power law for this metric) is larger than the 625 threshold (0.1) needed to accept the power law hypothesis (Clauset et al., 2009).

\section{Derivation of power law scaling of ORN ensemble responses from $E C_{50}$ distribution} Here, we explain analytically the power law relation between odorant concentration and the ensemble response of ORNs. Under the same Hill equation we used to fit individual doseresponse curves (Eq. 1, here we set $y_{\max }=1$ for simplicity), assume that (i) $E C_{50}$ follows a

631 power law distribution $P\left(1 / E C_{50}\right) \propto\left(1 / E C_{50}\right)^{-\lambda-1}$ (or equivalently an exponential distribution for $632 k=-\ln \left(E C_{50}\right): P(k)=\lambda \mathrm{e}^{-\lambda\left(k-k_{0}\right)}, k \geq k_{0}$ ) (ii) the Hill coefficient $n$ for all odorant-ORN pairs are 633 the same and greater than $\lambda$ (satisfied in the data as $1.45 \mathrm{vs.} 0.35$ ). If so, the ensemble 634 response follows an approximate power law form $r(c) \propto c^{\lambda}$ for concentrations $c \leq e^{-k_{0}}$ (which 635 means the weakest response pair in the ensemble has not reached the half level). For 
636

637

638

639

640

641

642

643

644

645

646

647

648

649

650

651

652

653

654

655

656

For the general case of logistic activation, the integral does not have a simple form expression but involves hyper-geometric functions. However, we can derive a simple closed form approximation by approximating the logistic function $f(x)=1 /\left(1+e^{-n x}\right)$ using piecewise exponential functions: logistic function in place of the Hill equation: $y(x)=\frac{1}{1+\exp (-n(x+k))}$.

This result can be intuitively obtained by considering the limiting case where the logistic function is infinitely steep (large Hill coefficient) and is thus replaced by a step function. The ensemble response combining a large number of odorant-ORN pairs can be expressed as an integral:

$r(x)=\int_{k_{0}}^{\infty} y(x, k) \lambda e^{-\lambda\left(k-k_{0}\right)} d k, y(x, k)=\frac{1}{1+\exp (-n(x+k))}$ is the log-concentration. When $y(x, k)$ is a step function, the integral becomes $P(k \geq-x)$, which is essentially the cumulative density function for $k$. Given the distribution of $k$, this is exactly an exponential function $r(x)=e^{\lambda\left(x+k_{0}\right)}$ (or a power law function of $c$ ) for $x \leq-k_{0}$, and saturates at larger concentrations.

Such an approximation becomes asymptotically exact when the steepness $n$ goes to infinity, or when the absolute value of $x$ goes to infinity. Substituting $y(x, k)$ with this approximation, the integral splits into segments, over which the integrand are sums of exponential functions, and therefore can be easily integrated. This gives the closed form approximation of $r(x)$ : $r(x)=\left\{\begin{array}{c}\left(1+\frac{2 \lambda^{2}}{n^{2}-\lambda^{2}}-\frac{\lambda^{2}}{4 n^{2}-\lambda^{2}}\right) e^{\lambda\left(x+k_{0}\right)}-\frac{\lambda}{n-\lambda} e^{n\left(x+k_{0}\right)}+\frac{\lambda}{2(2 n-\lambda)} e^{2 n\left(x+k_{0}\right)}, x \leq-k_{0} \\ 1-\frac{\lambda}{n+\lambda} e^{-n\left(x+k_{0}\right)}+\frac{\lambda}{2(2 n+\lambda)} e^{-2 n\left(x+k_{0}\right)}, x>-k_{0}\end{array}\right.$ For small concentrations, $x \leq-k_{0}$, the leading term in the above expression is $e^{\lambda\left(x+k_{0}\right)}$, since $\lambda<n$. This explains that the ensemble response is approximated by an exponential function with exponent $\lambda$. Furthermore, the theory also predicts the magnitude (vertical shift in the log-log 662 plot of ensemble response as in Fig $3 \mathbf{C})$, that is, $r(x) \approx\left(1+\frac{7}{4} \frac{\lambda^{2}}{n^{2}}\right) e^{\lambda\left(x+k_{0}\right)}$, which explains how the Hill coefficient affect the ensemble response. 
664 White noise experiments were performed in a manner similar to those described in Kato, et. al,

665 (2014). Briefly, we used custom code written in MATLAB to control odorant and water switching

666 such that it followed an m-sequence. Calcium imaging was performed on the axon terminal of

667 individual ORNs at $\sim 30$ frames per second. Calibration and an example of such a recording is

668 shown in Movie 2. We then used a linear-nonlinear model to compare the $m$-sequence input to

669 ORN responses during a 150second interval (from $60-210 \mathrm{sec}$ ). An 18 second time window

670 was used for the linear filter, of which 15 seconds represented stimulus history in order to

671 ensure extraction of the full filter dynamics (Supp Fig 7B). Next, we applied the linear filter to

672 the data and compared this to the output in order to capture the nonlinear function. We found

673 that a sigmoidal function fits the nonlinear function well (Supp Fig 7C). We applied novel m-

674 sequences to validate the linear-nonlinear model (Supp Fig 7D) and found that they fit the data

675 well. Peak and decay times for each filter were found by extracting the time points

676 corresponding to the maximum amplitude and half maximum amplitude of the decay phase,

677 respectively. 454 filters were calculated from the recording of 138 larvae responding to various

$678 \mathrm{~m}$-sequence stimuli. Each of the 31 filters quantified in Supp Fig 7, are averaged across 10

679 trials.

680

681 Data, code and software can be found at: https://github.com/samuellab/larval olfaction

682 Microfluidic device pattern design can be found at:

683 https://metafluidics.org/devices/larval olfaction/ 


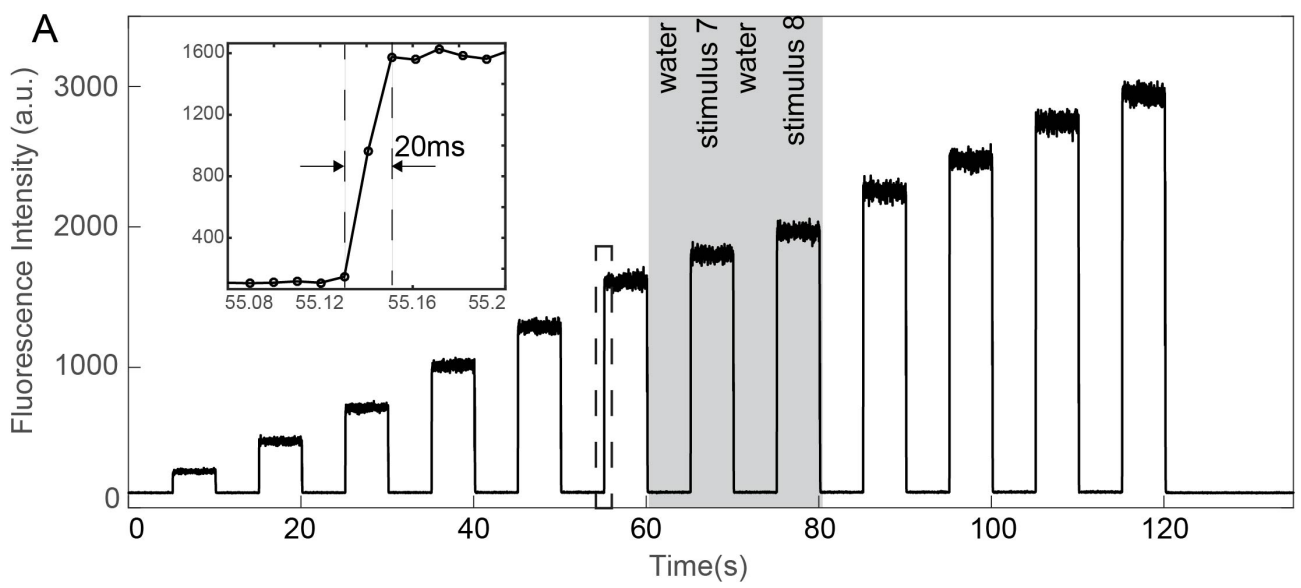

B $\begin{array}{lllllllllll}\mathrm{C}_{\mathrm{c} 1} & \mathrm{C}_{\mathrm{w}} & \mathrm{C}_{\mathrm{s} 1} & \mathrm{C}_{\mathrm{s} 2} & \ldots & \mathrm{C}_{\mathrm{s} 7} & \mathrm{Cs}_{\mathrm{s} 8} & \ldots & \mathrm{C}_{\mathrm{s} 12} & \mathrm{C}_{\mathrm{s} 13} & \mathrm{C}_{\mathrm{c} 2}\end{array}$

$\begin{array}{rrrrrrrrrrrr}\text { water } & 1 & 1 & 0 & 0 & \ldots & 1 & 0 & \ldots & 0 & 0 & 0 \\ \text { stimulus } 7 & 0 & 1 & 0 & 0 & \ldots & 1 & 0 & \ldots & 0 & 0 & 1 \\ \text { water } & 1 & 1 & 0 & 0 & \ldots & 0 & 1 & \ldots & 0 & 0 & 0 \\ \text { stimulus 8 } & 0 & 1 & 0 & 0 & \ldots & 0 & 1 & \ldots & 0 & 0 & 1\end{array}$

C

water

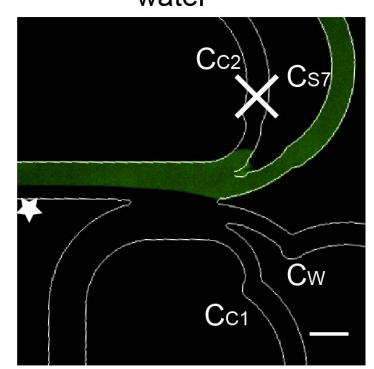

stimulus 7

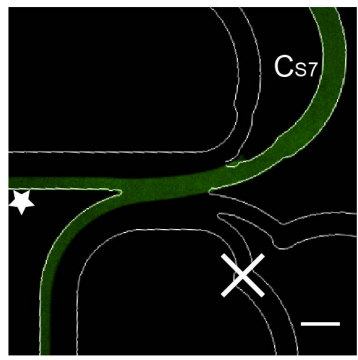

water

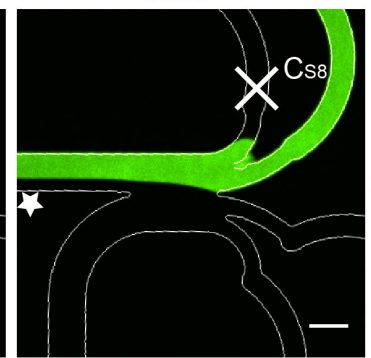

stimulus 8

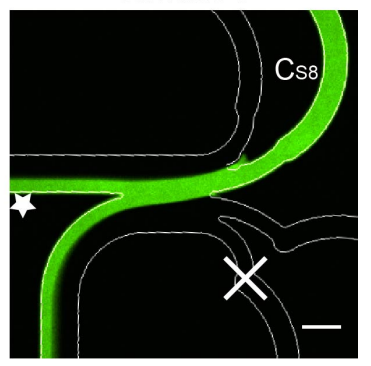

Supplementary Figure 1. Validation of temporal and spatial odorant profiles in

microfluidics device, using a fluorescent dye. A. Change in fluorescence intensity during

delivery of $5 \mathrm{sec}$ step pulses of increasing concentration of fluorescein dye, each followed by 5 second of water. Inset shows zoom-in of dashed box, indicating stimulus transition time is $\sim 20$ ms. B. Combination of valve states required to generate the stimulation sequence in shaded area of panel $A ; 1$ and 0 indicate valve is open or closed, respectively. $C_{W}$ represents water channel, $\mathrm{C}_{\mathrm{C} 1}$ and $\mathrm{C}_{\mathrm{C} 2}$ represent control channels that allow stimulus switching, and $\mathrm{C}_{7}$ and $\mathrm{C}_{8}$ represent odorant delivery channels and only open prior to and during stimulus delivery. C. Images of fluorescein dye, representing an odorant stimulus, in the microfluidics device during each state shown in panel B (water, stimulus 7, stimulus 8). Cross mark indicates closed channels, star marks the location of the larva's "nose". Scale bar is $300 \mu \mathrm{m}$. 
A
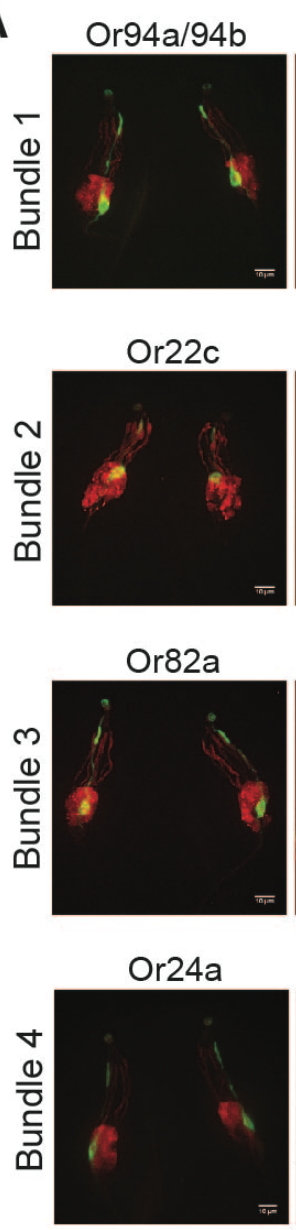

Or49a

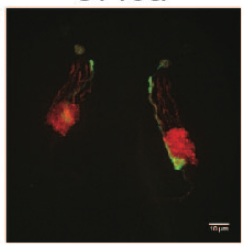

Or42b

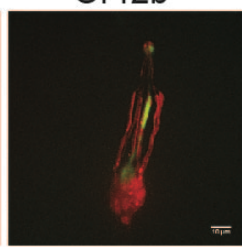

Or13a

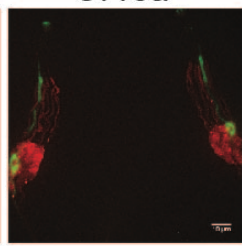

Or85c

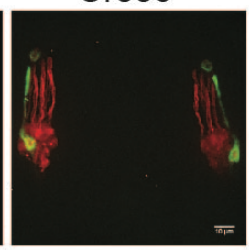

Left

Dorsal Organ

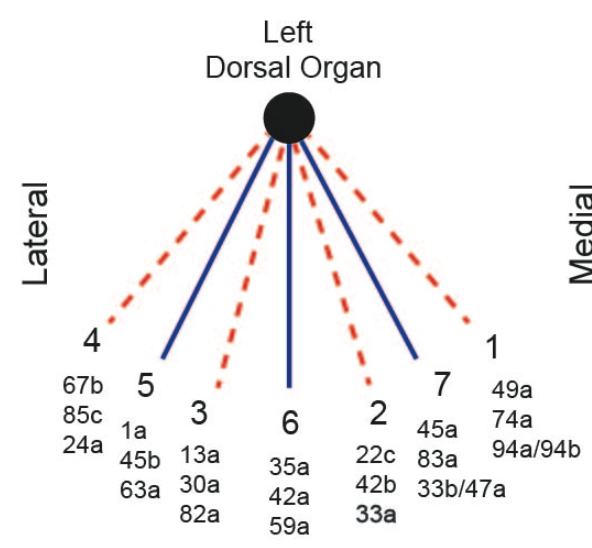

696

B

Posterior

Or30a

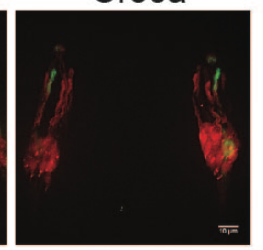

Or67b

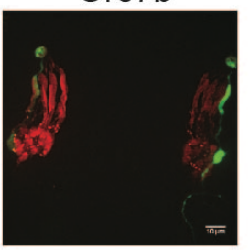

Anterior

$\frac{\bar{c}}{\overline{0}}$
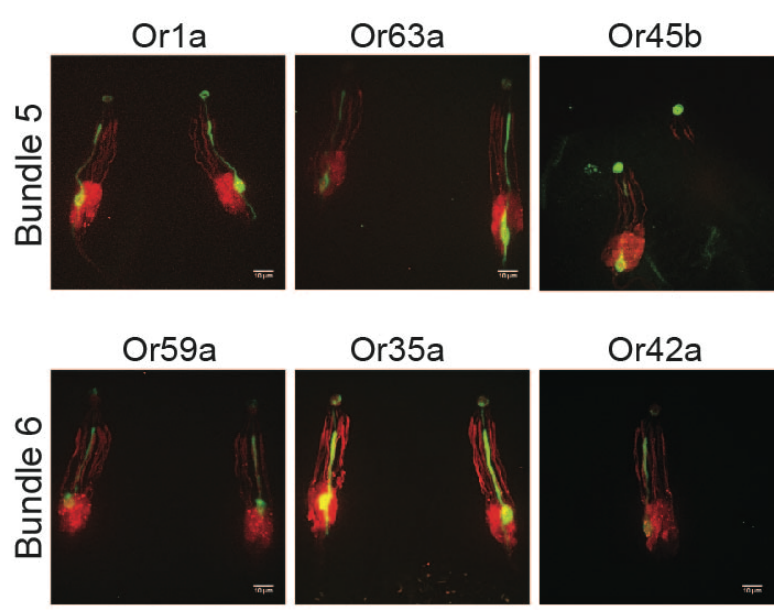

Or35a

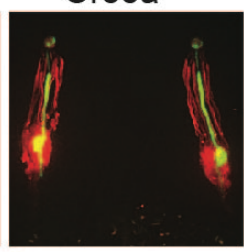

Or42a
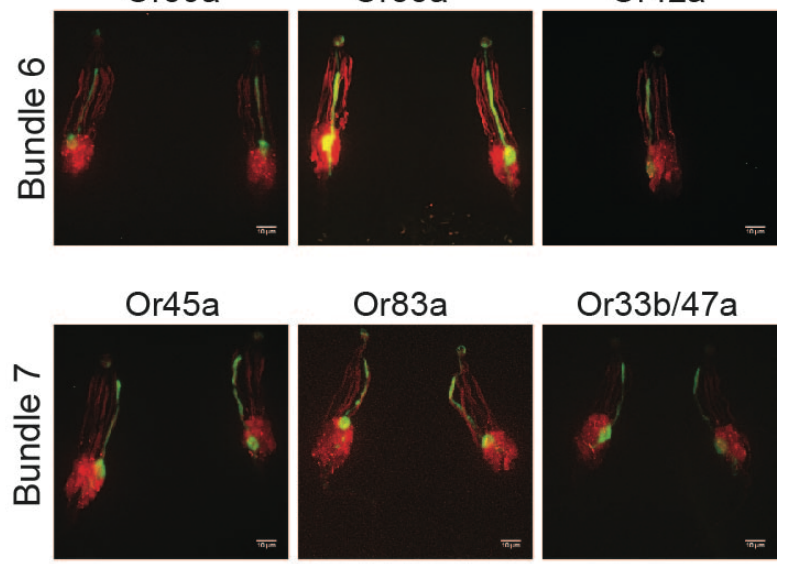

Or83a

Or33b/47a
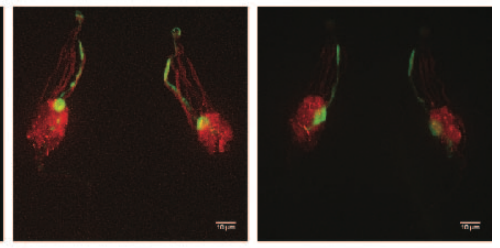

Right

Dorsal Organ
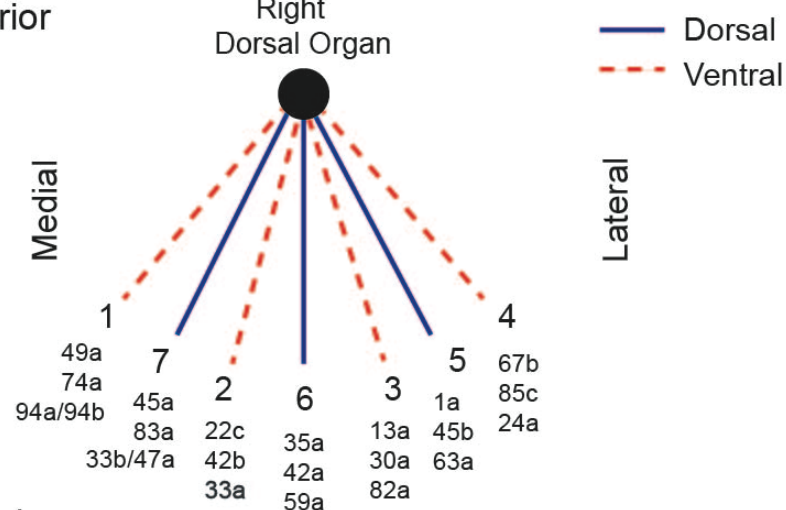

ত্

Supplementary Figure 2. Anatomical map of ORN dendritic organization. A. Image of each ORN's dendritic location using GFP to label a specific ORN and RFP to label all ORNs. Larvae expressing $\operatorname{Or} X>G F P$, Orco $>R F P$, where $\operatorname{OrX}$ is a specific olfactory receptor. We infer the vacancy in bundle 2 is Or33a. During functional imaging, there were no strong signals from this neuron. No expression of Or2a and Or7a were observed in first instar larvae. B. Summary 

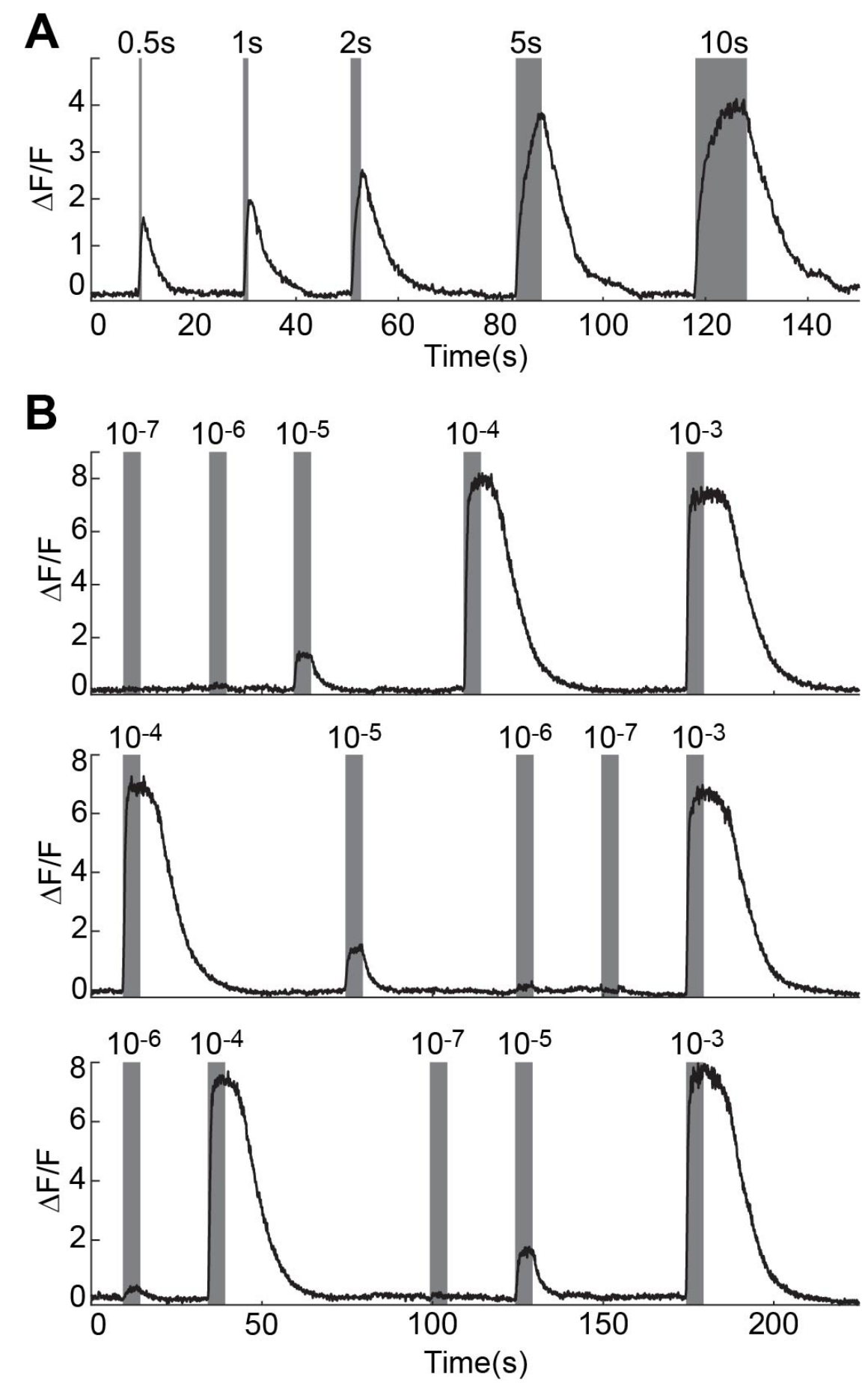

704 Supplementary Figure 3. Effect of stimulus duration and sequence on ORN response. A.

705 Or35a-ORN responses to $0.5,1,2,5$ and 10 seconds of $10^{-5}$ dilution of 3 -octanol. The

706 maximum response saturates when odorant pulse is longer than 5 seconds. B. Or35a-ORN

707 response to increasing (top panel), primarily decreasing (middle panel), and random (bottom

708 panel) concentration sequences of 3-octanol pulses, delivered at 5 seconds each. The response

709 amplitude to each concentration level is history independent. 


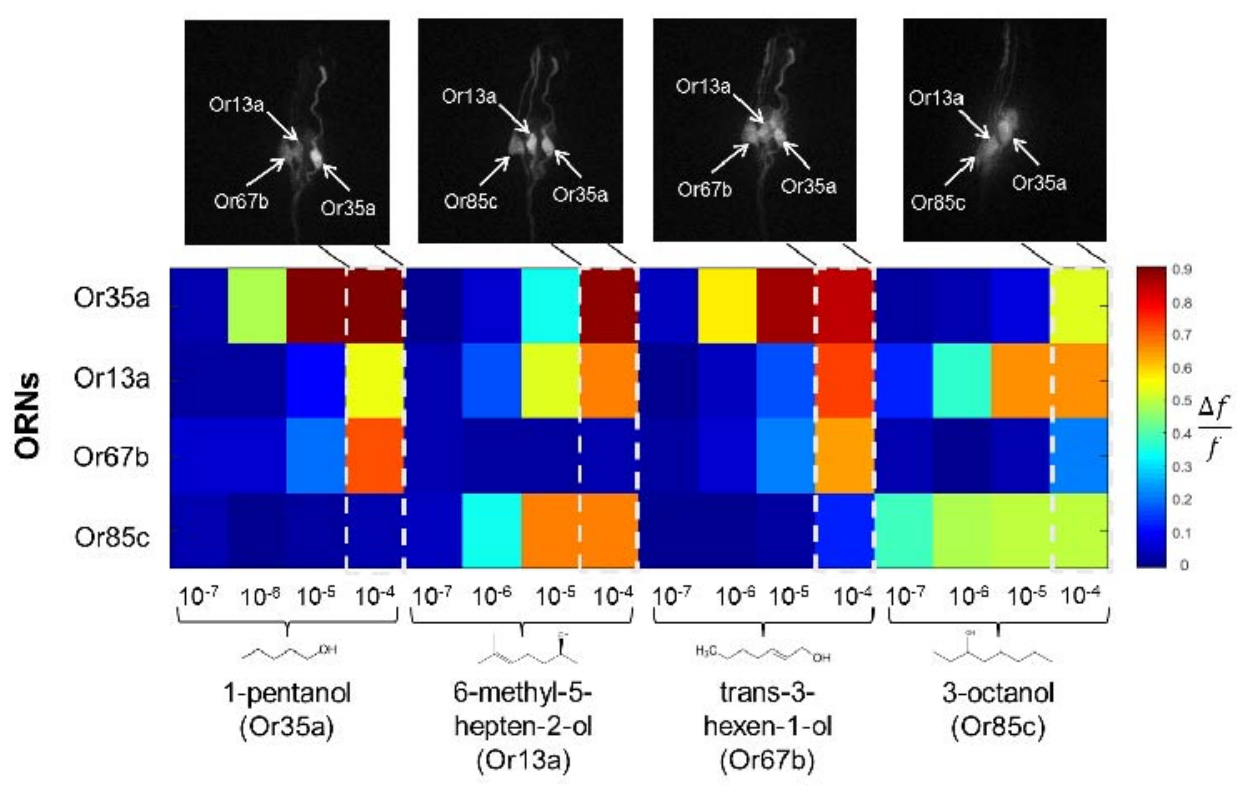

711 Supplementary Figure 4. Coactivation of ORNs with similar cognate odorants at high

712 concentrations. Heatmap of peak responses of four ORNs to four alcohol odorants, across

713 four concentrations of each odorant. Activities normalized by maximum response amplitude.

714 Neural images show responsive ORNs in dorsal organ ganglion during calcium imaging at the

715 highest odorant concentrations. 

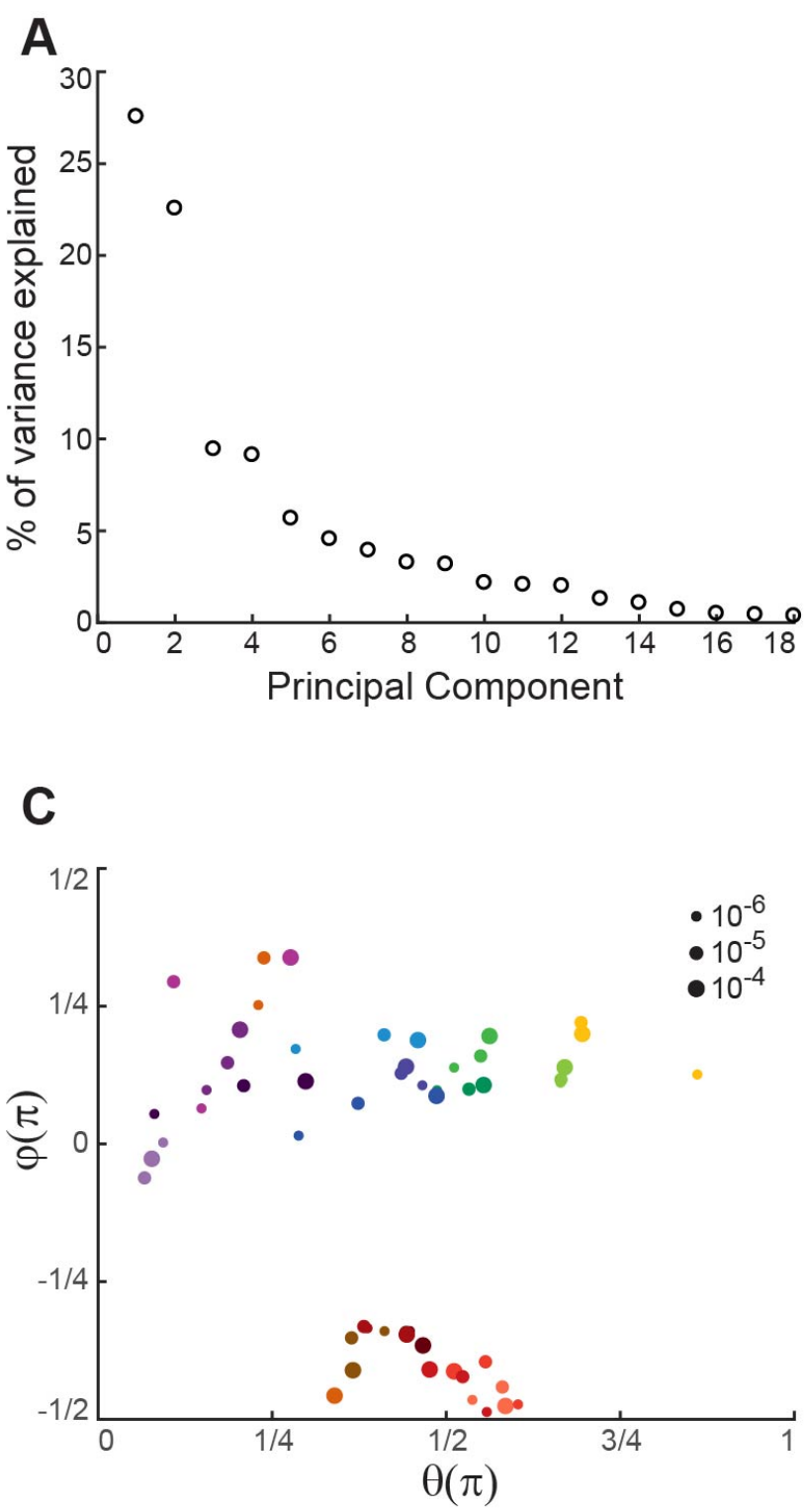

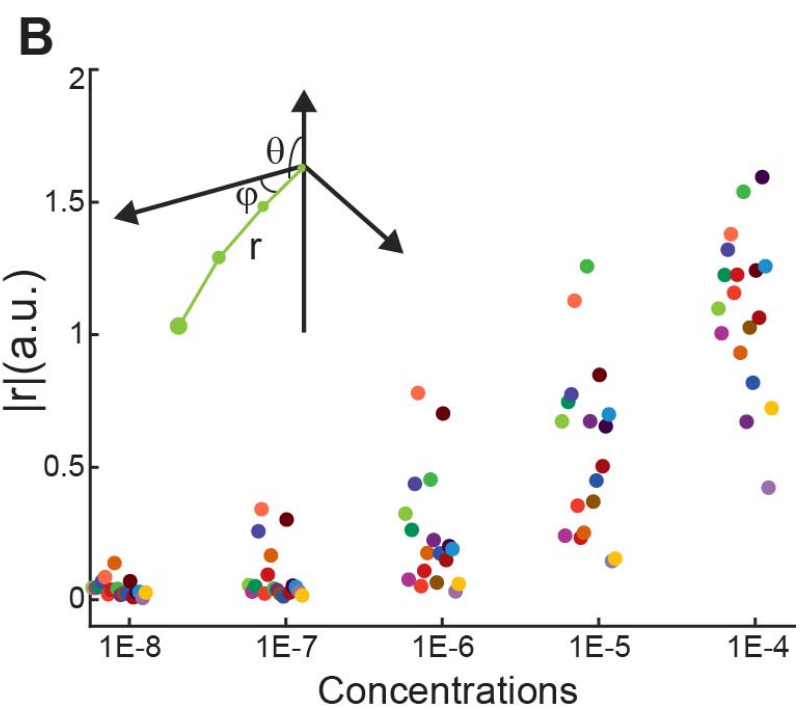

acetal

trans,trans-2,4-nonadienal

- 4-hexen-3-one

3-pentanol

- geranyl acetate

_ 6-methyl-5-hepten-2-ol

3-octanol

- 2-nonanone

pentyl acetate

1-pentanol

2-phenyl ethanol

2,5-dimethylpyrazine anisole methyl phenyl sulfide 2-acetylpyridine 4-methyl-5-vinylthiazole 4,5-dimethylthiazole 2-methoxyphenyl acetate

718 Supplementary Figure 5. PCA analysis of ORN dose-response data. A. Percentage of

719 variance explained by each principal component. B. and C. Transform of odorant vectors in

720 PCA space (Fig 2B) to spherical coordinates (inset of B). Odorant vector length increases

721 monotonically with increasing concentration. Angular direction of different odorants (represented

722 by dot color) separate, but aggregate for direction of different concentrations of the same

723 odorant (dot size). 

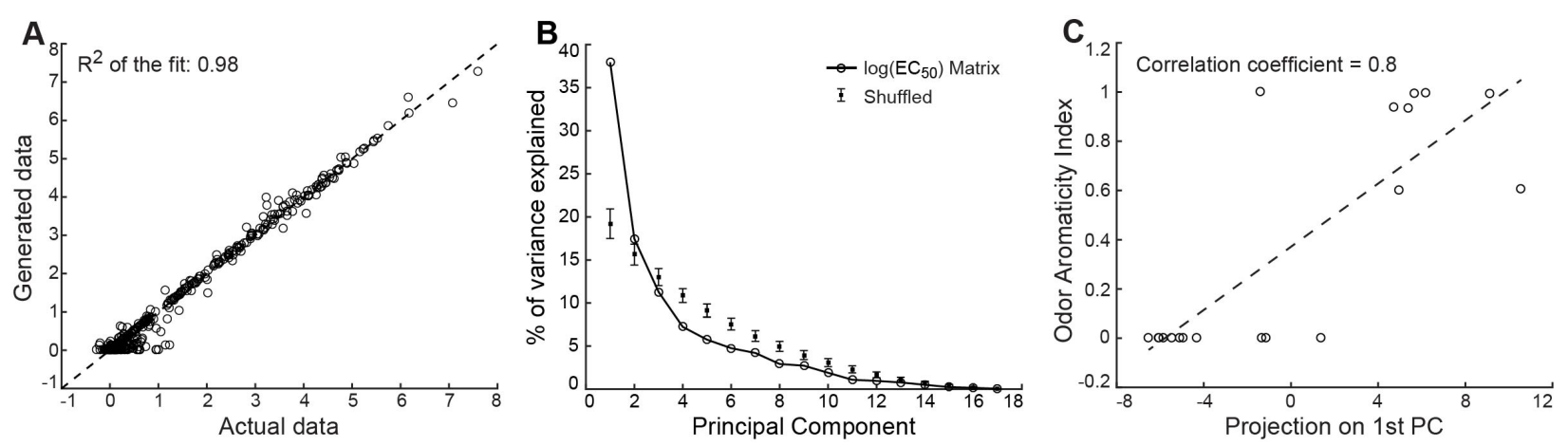

725 Supplementary Figure 6. Aromaticity index highly correlated to major PC of EC ${ }_{50}$ matrix.

726 A. Actual neural activity data is highly correlated with simulated data generated using the Hill

727 equation and fitted parameters. Scatter plot includes all non-zero data in Fig 2A. Dashed line

728 indicates $y=x \cdot R^{2}$ is 0.98 . B. Percentage of variance explained by each PC of the PCA on the

$729-\ln \left(E C_{50}\right)$. Data compared with the corresponding results from 1000 randomly shuffled data. C.

730 Correlation plot between each odorants projection on the $1^{\text {st }} \mathrm{PC}$ of $-\ln \left(E C_{50}\right)$ matrix and its

731 aromaticity index. 
A

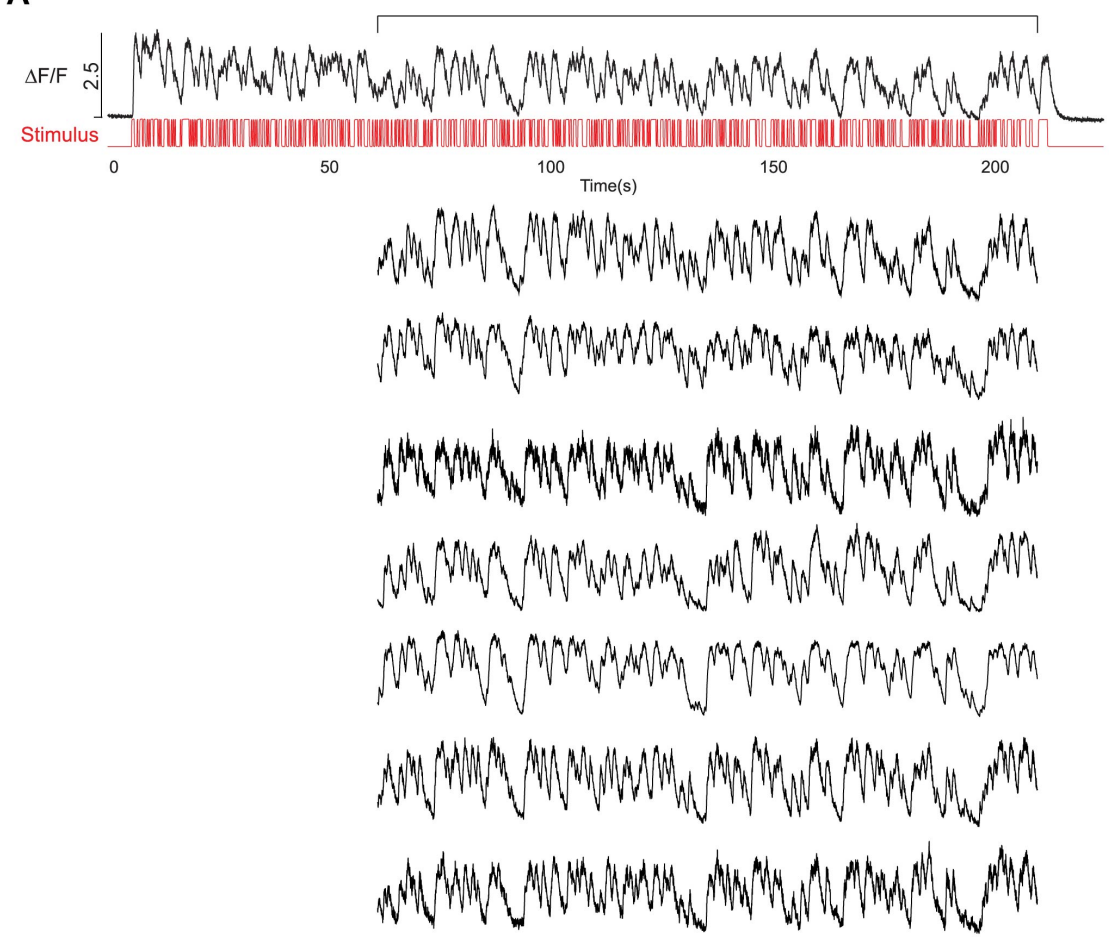

B

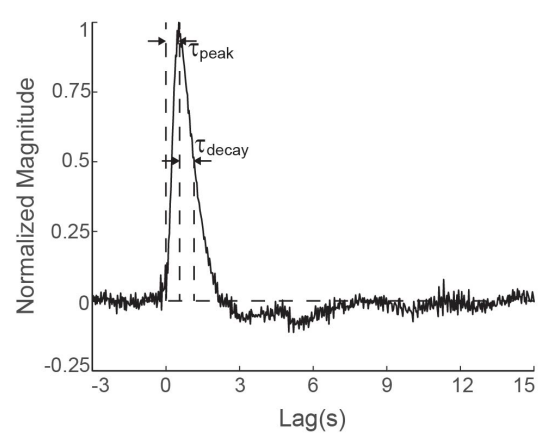

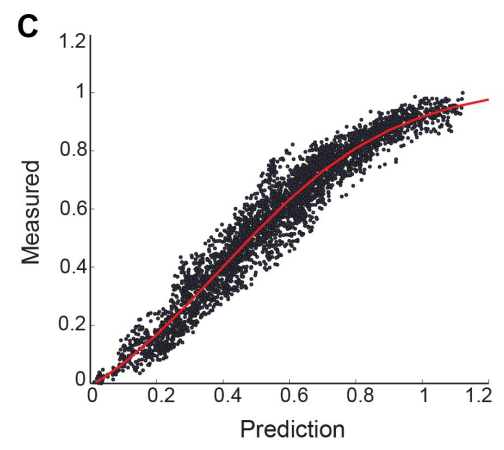

$$
\text { D }
$$

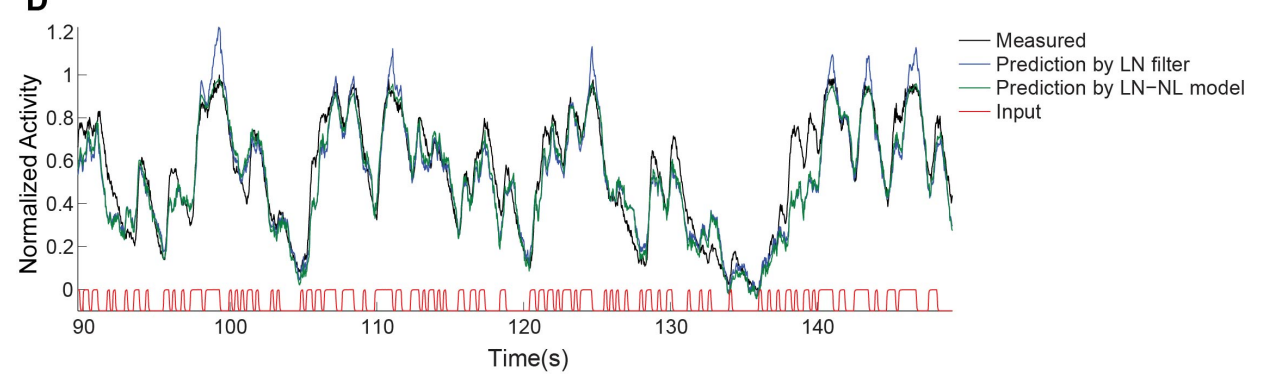

Supplementary Figure 7. Linear-nonlinear model of ORN temporal dynamics. A. Multiple

734 trial responses of Or42a-ORN to the same m-sequence of $10^{-7}$ dilution of 3-pentanol. B. The

735 linear filter calculated from reverse correlation of the input-output from the first trial of panel A. C.

736 Non-linear transfer function calculated by comparing measured and predicted responses using

737 the linear filter. D. Validation of the linear-nonlinear model by comparing predicted and

738 measured responses to novel m-sequence stimuli. 
A

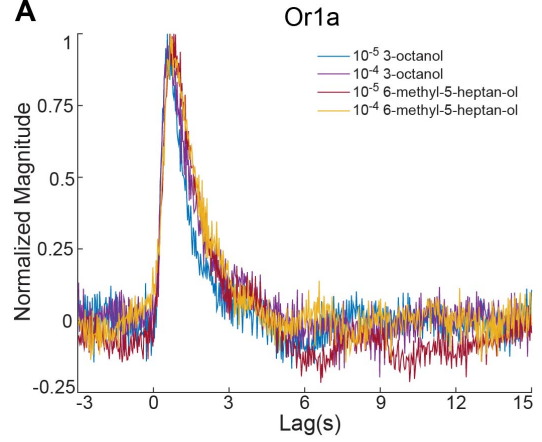

B

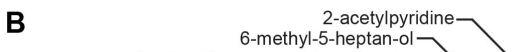

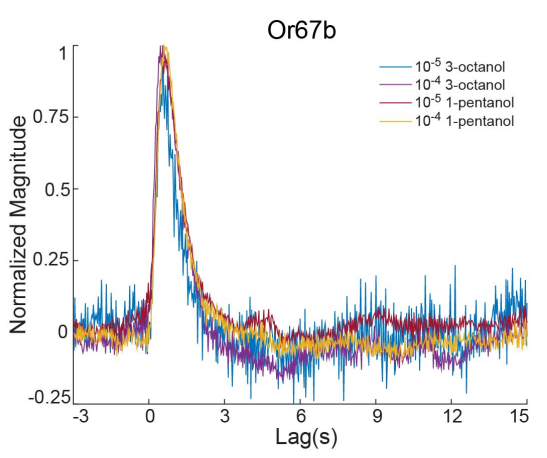

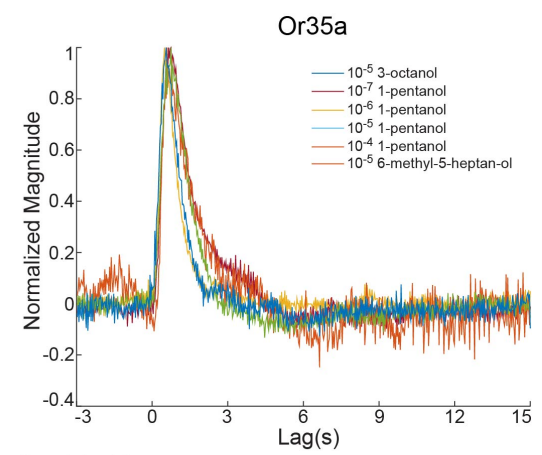

C

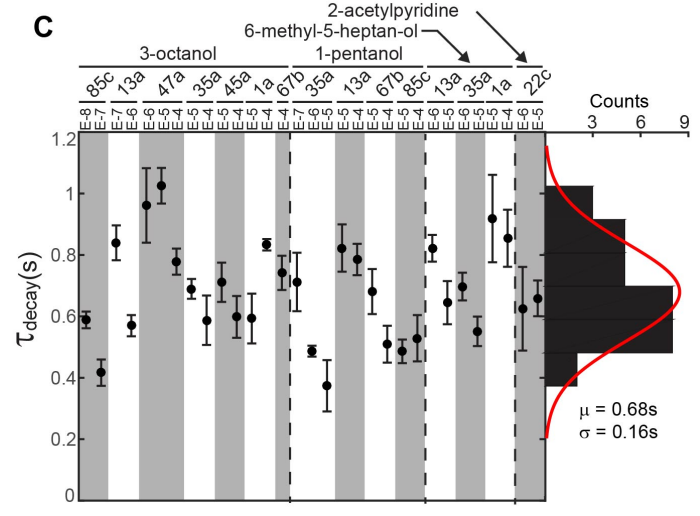

Supplementary Figure 8. Comparison of ORN temporal filters. A. Normalized filters of three ORNs responding to various odorant stimuli. B. and C. Distribution of peak time (B.) and decay time (C.) of 31 filters measured from various ORN and odorant stimuli. Distributions of peak and decay times were fit to Gaussian distributions with mean and variance labeled below histogram.

Movie 1. Dose-dependent activation of ORNs. Left: Calcium imaging of 21 pairs of larval ORNs in response to increasing concentrations of the 1-pentanol odorant, from $10^{-7}$ to $10^{-3}$ dilutions. Movie starts by scanning through the imaging volume to identify ORNs activated at the highest concentration level. Three ORNs are responsive on the left side and five on the right side. ORN identity was confirmed from the dendrite location and response to panel of 13 cognate odorants (not shown in the movie). Right: Responses of ORNs to step pulses of odorant stimuli.

Movie 2. ORN responses to pseudorandom white noise stimulus. Top left, stimulus delivery marked by fluorescence. Bottom left, axon terminal of Or45a-ORN responding to the white noise stimulus using $10^{-7}$ dilution of 2-nonanone. Top and bottom right, real time plots of the input stimulus and ORN response during an experiment. 
bioRxiv preprint doi: https://doi.org/10.1101/208538; this version posted October 25, 2017. The copyright holder for this preprint (which was not certified by peer review) is the author/funder, who has granted bioRxiv a license to display the preprint in perpetuity. It is made available under aCC-BY-NC-ND 4.0 International license.

758 Supplementary Table 1. Raw activity data of 21 ORNs responding to 19 odorants at five 759 concentration levels collected from 122 recordings. 
37. Anderson, J. R., Chiu, D. T., Wu, H., Schueller, O. J. \& Whitesides, G. M. Fabrication of microfluidic systems in poly (dimethylsiloxane). Electrophoresis, 21(1), 27-40 (2000).

38. Thevenaz, P., Ruttimann, U. E. \& Unser, M. A pyramid approach to subpixel registration based on intensity. IEEE transactions on image processing, 7(1), 27-41 (1998). Data. SIAM Review, 51(4), 661-703 (2009). 